\title{
Organo Phosphorus-Selenium Heteroatom Derivatives from Selenation of Primary/Secondary Amines and Haloalkanes/Dihaloalkanes
}

\author{
Guoxiong Hua, ${ }^{[a]}$ Kasun S. A. Arachchige, ${ }^{[a]}$ Alexandra M. Z. Slawin, ${ }^{[a]}$ and J. Derek Woollins ${ }^{*[a]}$
}

Keywords: Woollins' reagent / Selenation / Phosphorus-Selenium Compounds / Amines / Haloalkanes

Reaction of 2,4-bis(phenyl)-1,3-diselenadiphosphetane-2,4diselenide [Woollins' reagent, WR] with four equivalents of primary/secondary amines led to a series of ammonium phenylphosphonamidodiselenoates or phenylphosphonamidodiselenoic diamides 1a-n, the latter further reacted in situ with either two equivalents of haloalkanes or equimolar amount of dihaloalkanes resulting in the corresponding $S e$-alkylphenyl-
Se-alkylphenyl-phosphonamidodiselenoates 2a-n and alkane bis( $N$-alkyl- $P$-phenylphosphonamidodiselenoate)s 3a-n in good to excellent yields. All new compounds have been characterized spectroscopically including multi-NMR $\left({ }^{1} \mathrm{H},{ }^{13} \mathrm{C},{ }^{31} \mathrm{P}\right.$ and $\left.{ }^{77} \mathrm{Se}\right)$, IR and mass spectroscopy. Two representative $\mathrm{X}$-ray structures are also described.

\section{Introduction}

Organophosphorus chemistry has been attracted huge intention in both academic and industrial research for several decades. ${ }^{1-3} \mathrm{~A}$ wide range of applications have been documented in different fields such as coordination chemistry, material science, homogeneous catalysis, development of biologically active compounds or pesticides, and additives for polymers such as lubricants and antioxidants. ${ }^{4-10}$ 2,4-Bis(phenyl)-1,3diselenadiphosphethane-2,4-diselenide $\quad\left[\{\mathrm{PhP}(\mathrm{Se})(\mu-\mathrm{Se})\}_{2}\right]$ (Woollins' reagent, WR), a selenium counterpart of the wellknown Lawesson's reagent (LR), has been well developed as an efficient phosphorus-selenium containing building block or selenation reagent in organic synthetic chemistry in recent years. ${ }^{11}$ The reactions of WR with various organic substrates exhibit a diverse spectrum ranging from simple oxygen-selenium exchange to the formation of complex phosphorus-selenium heterocycles as well as the formation of surprising phosphorus-selenium-free products. ${ }^{12-25} \mathrm{We}$ recently have reported the synthesis of a series of ammonium phenylphosphonamidodiselenoates and phenylphosphonamidodiselenoic diamides from the reaction of WR with primary or secondary amines, ${ }^{26}$ and the formation of a series of organo phosphorus-selenium heteroatom derivatives from the selenation of alkenyl or arynyl-diamines. ${ }^{27}$ As a part of our continuous exploration into the reactivity of WR towards different organic nucleophiles, herein, we report the synthesis of a series of novel $S e$-alkylphenylphosphonamidodiselenoates and alkane bis $(N$ alkyl-P-phenylphosphonamidodiselenoate)s via the reaction of WR with primary/secondary amines, followed by the in situ treatment with haloalkanes or dihaloalkanes. We also report two representative $\mathrm{X}$-ray structures.

[a] EaStCHEM School of Chemistry, University of St Andrews, St Andrews, Fife, Scotland, KY16 9ST, UK Fax: +44-1334-463384

E-mail: jdw3@st-and.ac.uk. http://www.standrews.ac.uk/chemistry/

Supporting information for this article is available on the www under http://dx.doi.org/10.1002/ejoc.xxxxxxxxx.

\section{Results and Discussion}

Treatment of WR with four molar equivalents of primary or secondary amine in dry THF at ambient temperature for $2 \mathrm{~h}$ proceeded with the cleavage of the four-membered ring in WR leading to the corresponding ammonium salts of phenylphosphonamidodiselenoates and phenylphosphonamidodiselenoic diamides 1a-n in almost quantitative yields. ${ }^{26}$ These ammonium salts were further reacted in situ with two equivalents of haloalkanes at ambient temperature for $24 \mathrm{~h}$ to give the corresponding $\mathrm{Se}$ alkylphenylphosphonamidodiselenoates $\mathbf{2 a - n}$ as depicted in Scheme 1 . These new heteroatom compounds were isolated in good to excellent yields $(41 \%-93 \%)$ (Table 1) as sticky oils or pastes which are soluble in both chlorinated solvents and tetrahydrofuran. It should be noted that products $2 \mathrm{e}-\mathrm{I}$ bearing the strong electron-withdrawing group $\mathrm{R}^{3}\left(\mathrm{R}^{3}=p-\mathrm{BrC}_{6} \mathrm{H}_{4} \mathrm{COCH}_{2}\right)$ were obtained in rather high yields $(55 \%-93 \%)$, meanwhile, products $\mathbf{2 b}$ and $\mathbf{2 c}$ bearing less electron-withdrawing group $\mathrm{R}^{3}\left(\mathrm{R}^{3}\right.$ $=\mathrm{CH}_{3}\left(\mathrm{CH}_{2}\right)_{10}$ or $\left.\mathrm{CH}_{3}\left(\mathrm{CH}_{2}\right)_{6}\right)$ were isolated in the lowest yields $(41 \%$ and $42 \%)$. Thus, it appears that a strong electronwithdrawing group $\mathrm{R}^{3}$ is hugely beneficial for the formation of the reaction products. Compounds $\mathbf{2 a - n}$ are stable to air or moisture for months without any obvious signals of degradation.

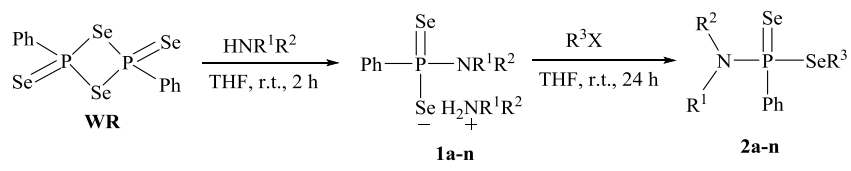

Scheme 1 Synthesis of $S e$-alkylphenylphosphonamidodiselenoates 2a-n (the $\mathrm{R}$ groups are defined in Table 1 below)

2a-n were characterised by ${ }^{1} \mathrm{H},{ }^{13} \mathrm{C},{ }^{31} \mathrm{P}$ and ${ }^{77} \mathrm{Se} \mathrm{NMR}$, IR spectroscopy and mass spectrometry. All new compounds showed the anticipated $[\mathrm{M}+\mathrm{H}]^{+}$or $[\mathrm{M}+\mathrm{Na}]^{+}$peak in their mass spectra and satisfactory accurate mass measurements. Two isomers were found in the NMR spectra of $\mathbf{2 f}$. The ${ }^{31} \mathrm{P}$ NMR spectra of $\mathbf{2 a - n}$ display sharp singlets in the range of $46.9-78.4 \mathrm{ppm}$, flanked by two pairs of selenium satellites with ${ }^{31} \mathrm{P}_{-}{ }^{77} \mathrm{Se}$ coupling constants in the range 
of $355-391 \mathrm{~Hz}$ and $777-798 \mathrm{~Hz}$ indicating the presence of P-Se single bonds and $\mathrm{P}=\mathrm{Se}$ double bonds in each compound. This was further substantiated by the ${ }^{77} \mathrm{Se}$ NMR spectra of 2a-n which contain signals arising from SeR in the range of $\delta=299.8-384.0$ $\mathrm{ppm}$ and from the $\mathrm{P}=\mathrm{Se}$ selenium in the range of $\delta=-104.8--26.1$ ppm, respectively with matching ${ }^{31} \mathrm{P}_{-}{ }^{77} \mathrm{Se}$ coupling constants as shown in Table 1.

Table $1 S e$-alkylphenylphosphonamidodiselenoates 2a-n and their ${ }^{31} \mathrm{P}$ and ${ }^{77} \mathrm{Se} \mathrm{NMR}$ spectra

\begin{tabular}{|c|c|c|c|c|c|c|c|}
\hline $\mathrm{X}$ & $\mathrm{R}^{1}$ & $\mathrm{R}^{2}$ & $\mathrm{R}^{3}$ & $\begin{array}{l}\text { Pro } \\
\text { duct }\end{array}$ & $\begin{array}{l}\text { Yield } \\
(\%)\end{array}$ & $\begin{array}{l}\delta_{\mathrm{P}}, \mathrm{ppm} \\
{\left[\left(J_{\mathrm{P}, \mathrm{Se}}\right),\right.} \\
\mathrm{Hz}]\end{array}$ & $\begin{array}{r}\delta_{\mathrm{Se}}, \mathrm{ppm} \\
{\left[\left(J_{\mathrm{P}, \mathrm{Se}}\right), \mathrm{Hz}\right]}\end{array}$ \\
\hline $\mathrm{Cl}$ & $\begin{array}{c}c- \\
\text { Hexyl }\end{array}$ & $\mathrm{H}$ & $\mathrm{C}_{6} \mathrm{H}_{5} \mathrm{COCH}_{2}$ & $2 a$ & 54 & $\begin{array}{c}53.7 \\
{[371 / 792]}\end{array}$ & $\begin{array}{l}345.4[371] \\
-102.0[792]\end{array}$ \\
\hline $\mathrm{Br}$ & $\begin{array}{c}c^{-} \\
\text {Hexyl }\end{array}$ & $\mathrm{H}$ & $\mathrm{CH}_{3}\left(\mathrm{CH}_{2}\right)_{10}$ & $2 \mathrm{~b}$ & 42 & $\begin{array}{c}53.2 \\
{[391 / 786]}\end{array}$ & $\begin{array}{l}323.6[391] \\
-104.5[786]\end{array}$ \\
\hline $\mathrm{Br}$ & $\begin{array}{c}c^{-} \\
\text {Hexyl }\end{array}$ & $\mathrm{H}$ & $\mathrm{CH}_{3}\left(\mathrm{CH}_{2}\right)_{8}$ & $2 \mathrm{c}$ & 41 & $\begin{array}{c}53.2 \\
{[390 / 786]}\end{array}$ & $\begin{array}{l}323.6[390] \\
-104.6[786]\end{array}$ \\
\hline $\mathrm{Br}$ & $\begin{array}{c}c- \\
\text { Hexyl }\end{array}$ & $\mathrm{H}$ & $\mathrm{CH}_{3}\left(\mathrm{CH}_{2}\right)_{6}$ & $2 d$ & 59 & $\begin{array}{c}53.2 \\
{[390 / 786]}\end{array}$ & $\begin{array}{c}323.6[391] \\
-104.4[786]\end{array}$ \\
\hline $\mathrm{Br}$ & 'Bu & $\mathrm{H}$ & $\begin{array}{c}1,4- \\
\mathrm{BrC}_{6} \mathrm{H}_{4} \mathrm{COCH}_{2}\end{array}$ & $2 \mathrm{e}$ & 82 & $\begin{array}{c}46.9 \\
{[355 / 794]}\end{array}$ & $\begin{array}{l}384.0[355] \\
-53.0[792]\end{array}$ \\
\hline $\mathrm{Br}$ & ${ }^{\mathrm{s}} \mathrm{Bu}$ & $\mathrm{H}$ & $\begin{array}{c}1,4- \\
\mathrm{BrC}_{6} \mathrm{H}_{4} \mathrm{COCH}_{2}\end{array}$ & $2 f^{*}$ & 93 & $\begin{array}{c}56.0 \\
{[366 / 796]} \\
54.8 \\
{[373 / 782]}\end{array}$ & $\begin{array}{c}346.1[367] \\
-101.9[794] \\
348.5[374] \\
-104.8[784]\end{array}$ \\
\hline $\mathrm{Br}$ & ${ }^{n} \mathrm{Bu}$ & $\mathrm{H}$ & $\begin{array}{c}1,4- \\
\mathrm{BrC}_{6} \mathrm{H}_{4} \mathrm{COCH}_{2}\end{array}$ & $2 \mathrm{~g}$ & 81 & $\begin{array}{c}58.0 \\
{[370 / 794]}\end{array}$ & $\begin{array}{l}321.2[370] \\
-100.4[794]\end{array}$ \\
\hline $\mathrm{Br}$ & $\begin{array}{c}c- \\
\text { Hexyl }\end{array}$ & $\mathrm{H}$ & $\begin{array}{c}1,4- \\
\mathrm{BrC}_{6} \mathrm{H}_{4} \mathrm{COCH}_{2}\end{array}$ & $2 \mathrm{~h}$ & 56 & $\begin{array}{c}54.5 \\
{[369 / 794]}\end{array}$ & $\begin{array}{c}347.7 \text { [369] } \\
-100.7 \text { [794] }\end{array}$ \\
\hline $\mathrm{Br}$ & $\begin{array}{c}c- \\
\text { Pentyl }\end{array}$ & $\mathrm{H}$ & $\begin{array}{c}1,4- \\
\mathrm{BrC}_{6} \mathrm{H}_{4} \mathrm{COCH}_{2}\end{array}$ & $2 \mathbf{i}$ & 81 & $\begin{array}{c}55.8 \\
{[369 / 791]}\end{array}$ & $\begin{array}{r}338.8[369] \\
-95.3[791]\end{array}$ \\
\hline $\mathrm{Br}$ & ${ }^{1} \mathrm{Pr}$ & $\mathrm{H}$ & $\begin{array}{c}1,4- \\
\mathrm{BrC}_{6} \mathrm{H}_{4} \mathrm{COCH}_{2}\end{array}$ & $2 \mathrm{j}$ & 88 & $\begin{array}{c}54.6 \\
{[369 / 794]}\end{array}$ & $\begin{array}{c}345.3[370] \\
-102.4[794]\end{array}$ \\
\hline $\mathrm{Br}$ & ${ }^{\mathrm{i}} \mathrm{Pr}$ & $\mathrm{P} P \mathrm{Pr}$ & $\begin{array}{c}1,4- \\
\mathrm{BrC}_{6} \mathrm{H}_{4} \mathrm{COCH}_{2}\end{array}$ & $2 \mathrm{k}$ & 55 & $\begin{array}{c}62.5 \\
{[366 / 789]}\end{array}$ & $\begin{array}{l}311.7[367] \\
-26.1[789]\end{array}$ \\
\hline $\mathrm{Br}$ & ${ }^{i} \mathrm{Bu}$ & $\mathrm{Bu}$ & $\begin{array}{c}1,4- \\
\mathrm{BrC}_{6} \mathrm{H}_{4} \mathrm{COCH}_{2}\end{array}$ & 21 & 67 & $\begin{array}{c}78.4 \\
{[362 / 798]}\end{array}$ & $\begin{array}{l}299.8[362] \\
-93.5[799]\end{array}$ \\
\hline $\mathrm{Br}$ & ${ }^{1} \mathrm{Pr}$ & $\mathrm{Pr}$ & $\begin{array}{c}1,2- \\
\left(\mathrm{HOCH}_{2}\right) \mathrm{C}_{6} \mathrm{H}_{4} \\
\mathrm{CH}_{2}\end{array}$ & $2 \mathrm{~m}$ & 44 & $\begin{array}{c}59.9 \\
{[387 / 777]}\end{array}$ & $\begin{array}{r}361.4[388] \\
-26.4[777]\end{array}$ \\
\hline $\mathrm{Br}$ & $\mathrm{B}_{\mathrm{n}}$ & $\mathrm{H}$ & $\begin{array}{c}1,4- \\
\mathrm{BrC}_{6} \mathrm{H}_{4} \mathrm{COCH}_{2}\end{array}$ & $2 n$ & 47 & $\begin{array}{c}58.3 \\
{[376 / 798]}\end{array}$ & $\begin{array}{c}324.8[376] \\
-103.4[798]\end{array}$ \\
\hline
\end{tabular}

* Two diastereomers were found in compound $\mathbf{2 f}$.

The molecular structures of $\mathbf{2 e}$ and $\mathbf{2} \mathbf{i}$ were confirmed by singlecrystal X-ray diffraction studies as shown in Figures $1^{28}$ and $2 .^{29}$ The transparent, and colourless cubic crystals of $2 \mathbf{e}$ and $\mathbf{2 i}$ were grown from dichloromethane solution with slow diffusion of hexane. Compound $\mathbf{2 e}$ crystallizes in the triclinic space group $P-1$, meanwhile, compound $\mathbf{2} \mathbf{i}$ in the monoclinic space group $P 2_{1 / c}$ with two independent molecules per unit cell. In compound $2 \mathbf{e}$ the two phenyl rings are inclined $56.85^{\circ}$ to one another. The $\mathrm{P}(1)-\mathrm{Se}(1)$ of 2.103(3) $\AA$ and $\mathrm{P}(1)-\mathrm{Se}(2)$ bondlengths of 2.0954(13) $\AA$ in 2e and $\mathrm{P}(1)-\mathrm{Se}(1)$ of 2.097(4) [2.103(4)] and $\mathrm{P}(1)-\mathrm{Se}(2)$ of 2.281(4) [2.208(4)] $\AA$ in $\mathbf{2} \mathbf{i}$ are consistent with P-Se single bonds (2.2-2.3 $\AA$ ) and $\mathrm{P}=\mathrm{Se}$ double bonds $(2.08-2.12 \AA)$ in other similar compounds. $25,30,31$

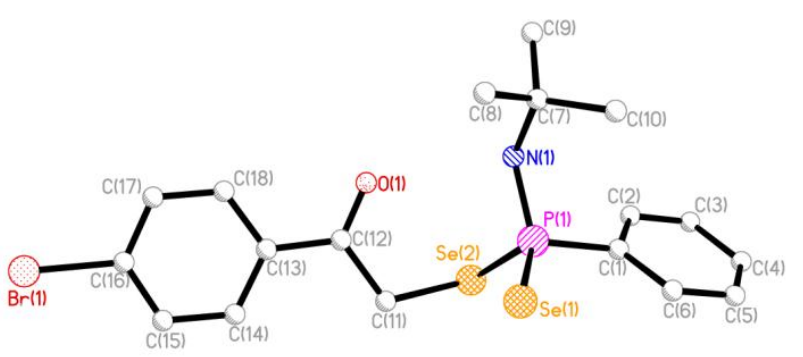

Figure 1. X-ray structure of compound 2e (Hydrogen atoms omitted for clarity). Selected bond lengths $(\AA)$ and angles $\left({ }^{\circ}\right)$ (esds in parentheses): $\mathrm{Se}(1)-\mathrm{P}(1)$ 2.103(3), $\mathrm{Se}(2)-\mathrm{P}(1)$ 2.257(3), $\mathrm{Se}(2)-$ $\mathrm{C}(11)$ 1.948(9), $\mathrm{P}(1)-\mathrm{C}(1)$ 1.807(8), $\mathrm{P}(1)-\mathrm{N}(1)$ 1.666(6), N(1)-C(7) 1.488(9); $\mathrm{P}(1)-\mathrm{Se}(2)-\mathrm{C}(11)$ 99.3(3), $\mathrm{Se}(1)-\mathrm{P}(1)-\mathrm{Se}(2)$ 113.48(11), $\mathrm{Se}(1)-\mathrm{P}(1)-\mathrm{N}(1)$ 120.7(2), Se(1)-P(1)-C(1) 113.0(3), Se(2)-P(1)$\mathrm{N}(1)$ 100.8(3), Se(2)-P(1)-C(1) 100.4(3), N(1)-P(1)-C(1) 106.0(4), $\mathrm{P}(1)-\mathrm{N}(1)-\mathrm{C}(7)$ 124.9(5).

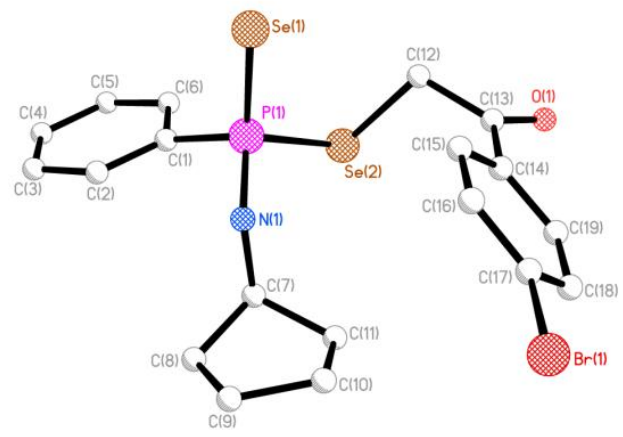

Figure 2. X-ray structure of compound 2i (Hydrogen atoms omitted for clarity). Selected bond lengths $(\AA)$ and angles $\left(^{\circ}\right)$ (esds in parentheses) (Dimensions for second independent molecule in square parentheses): $\mathrm{Se}(1)-\mathrm{P}(1)$ 2.097(4) [2.103(4)], $\mathrm{Se}(2)-\mathrm{P}(1)$ 2.281(4) [2.208(4)], $\mathrm{Se}(2)-\mathrm{C}(12)$ 1.950(11) [1.972(10)], $\mathrm{P}(1)-\mathrm{C}(1)$ 1.791(10) [1.838(10)], $\mathrm{P}(1)-\mathrm{N}(1)$ 1.666(6) [1.644(9)], N(1)-C(7) 1.483(12) [1.513(12)]; P(1)-Se(2)-C(11) 99.4(3) [101.3(3)], $\mathrm{Se}(1)$ $\mathrm{P}(1)-\mathrm{Se}(2) \quad 114.09(12) \quad[114.29(12)], \quad \mathrm{Se}(1)-\mathrm{P}(1)-\mathrm{N}(1) \quad 113.3(3)$ [111.2 (4)], $\mathrm{Se}(1)-\mathrm{P}(1)-\mathrm{C}(1)$ 113.7(4) [113.3(4)], $\mathrm{Se}(2)-\mathrm{P}(1)-\mathrm{N}(1)$ 109.4(3) [110.1(3)], Se(2)-P(1)-C(1) 101.3(4) [100.6(4)], N(1)$\mathrm{P}(1)-\mathrm{C}(1)$ 106.4(5) [106.6(5)], $\mathrm{P}(1)-\mathrm{N}(1)-\mathrm{C}(7)$ 119.2(6) [121.1(7)].

We also carried out the analoguous reactions by using dihaloalkanes instead of mono-haloalkanes. Thus, ammonium phenyldiselenophosphonates 1a-n were further reacted in situ with one molar equivalent of dibromoalkanes in tetrahydrofuran at ambient temperature for $24 \mathrm{~h}$ to give the corresponding $\mathrm{Se}$ alkylphenylphosphonamidodiselenoates 3a-n, as shown in Scheme 2 . These new compounds were isolated in moderate to good yields (51 to $80 \%$ ) as listed in Table 2 as sticky oils or pastes or solids soluble in chloroform, dichloromethane and tetrahydrofuran, and insoluble in diethyl ether and hexane.

The characterisation of 3a-n was performed by ${ }^{1} \mathrm{H},{ }^{13} \mathrm{C},{ }^{31} \mathrm{P}$ and ${ }^{77} \mathrm{Se}$ NMR, IR spectroscopy and mass spectrometry. All new compounds showed the anticipated $[\mathrm{M}+\mathrm{H}]^{+}$or $[\mathrm{M}+\mathrm{Na}]^{+}$peak in their mass spectra and satisfactory accurate mass measurements were obtained for all compounds. The ${ }^{31} \mathrm{P}$ NMR spectra of $\mathbf{3 a}-\mathbf{h}, \mathbf{3} \mathbf{j}$ and $\mathbf{3 l}$ exhibit two sets of double resonances (as a consequence of the stereoisomers present) with two sets of satellites inasmuch, meanwhile, interestingly, the ${ }^{31} \mathrm{P}$ NMR spectra of $\mathbf{3 i}, \mathbf{3 m}$ and $\mathbf{3 n}$ display four sets of double resonances and three sets of double resonances was found in $\mathbf{3 k}$, as a consequence of the stereoisomers present (Table 2). Normally, two phosphorus atoms are potentially stereogenic centres in compounds 3a-n, thus, being stereotopic with $(R, R),(S, S),(S, R)$ and $(R, S)$ stereoisomers are possible. Not surprisingly, in the ${ }^{31} \mathrm{P}$ NMR spectra of $\mathbf{3 a}-\mathbf{h}, \mathbf{3 j}$ and $\mathbf{3 l}$ two 
phosphorus signals with different intensity ratio (see experimental section) were observed in the range of $\delta(\mathrm{p})=52.4-54.9 \mathrm{ppm}$, flanked by two sets of satellites for the endocyclic and exocyclic selenium atoms $\left[J\left(\mathrm{P}, \mathrm{Se}_{\text {indo }}\right)\right.$ coupling constants being in the range of $371-387 \mathrm{~Hz}$ and $J\left(\mathrm{P}, \mathrm{Se}_{\mathrm{exo}}\right)$ coupling constants in the range of $784-794 \mathrm{~Hz}$ ] though we are not able to assign them specifically to $(R, R),(S, S),(S, R)$ and $(R, S)$ stereoisomers. However, four phosphorus signals with very similar chemical shifts and different intensity ratio (see experiment section) were found in the ${ }^{31} \mathrm{P} N \mathrm{NM}$ spectra of compound $\mathbf{3 i}, \mathbf{3 m}$ and $\mathbf{3 n}$, meanwhile, three similar phosphorus signals with different intensity ratio (see experiment section) were also observed in the ${ }^{31} \mathrm{P}$ NMR spectrum of compound $\mathbf{3 k}$, and all of these signals were flanked by two sets of satellites for the endocyclic and exocyclic selenium atoms (see Table 2). The results indicate the presence of some spatial hindrance effects in compounds $\mathbf{3 i}, \mathbf{3 k}, \mathbf{3 m}$ and $\mathbf{3 n}$, compared with compounds $\mathbf{3 a - h}, \mathbf{3 j}$ and 3l. The hindered rotation of two big $\mathrm{Ph}\left(\mathrm{NR}^{1} \mathrm{R}^{2}\right) \mathrm{P}(\mathrm{Se}) \mathrm{CH}_{2}$ groups from ortho or meta position in the bridged linkage benzene backbone in compounds of $\mathbf{3 i}, \mathbf{3 k}, \mathbf{3} \mathbf{m}$ and $\mathbf{3 n}$ may result in three or four magnetically different phosphorus environments rather than three or four chemically different phosphorus environments when two potential stereogenic centers exist. Detailed ${ }^{31} \mathrm{P}$ and ${ }^{77} \mathrm{Se} \mathrm{NMR}$ spectroscopic analyses reveal the relatively small coupling constant ${ }^{3} J(\mathrm{P}, \mathrm{Se})=17.0 \mathrm{~Hz}$ and ${ }^{4} J(\mathrm{P}, \mathrm{P})=4.2 \mathrm{~Hz}$ in $\mathbf{3 a},{ }^{4} J(\mathrm{P}, \mathrm{Se})=12.3 \mathrm{~Hz}$ in $\mathbf{3 b}$ and $\mathbf{3 f}$, supporting the presence of the $\mathrm{P}(\mathrm{Se}) \mathrm{SeCH}_{2} \mathrm{SeP}(\mathrm{Se})$ or $\mathrm{P}(\mathrm{Se}) \mathrm{SeCH}_{2} \mathrm{CH}_{2} \mathrm{SeP}(\mathrm{Se})$ linkage in compounds $\mathbf{3 a}, \mathbf{3 b}$ and $3 \mathbf{f}$. The ${ }^{31} \mathrm{P}$ and ${ }^{77} \mathrm{Se}$ chemical shifts and coupling constants are comparable to phosphorus-selenium compounds in the literature. ${ }^{32-35}$

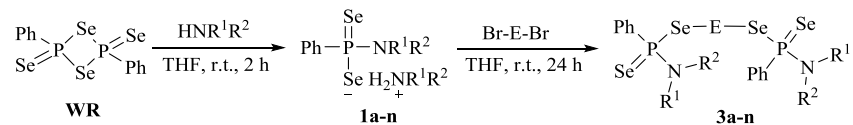

Scheme 2 Synthesis of $S e$-alkylphenylphosphonamidodiselenoates 3a-n (the $\mathrm{R}$ and $\mathrm{E}$ groups are defined in Table 2 below)

Table 2 Se-alkylphenylphosphonamidodiselenoates 3a-n and their

\begin{tabular}{|c|c|c|c|c|c|c|}
\hline $\begin{array}{l}\text { Pro } \\
\text { duct }\end{array}$ & $R^{1}$ & $\mathrm{R}^{2}$ & E & $\begin{array}{l}\text { Yield } \\
(\%)\end{array}$ & $\begin{array}{c}\delta_{\mathrm{P}, \mathrm{ppm}} \\
\left.\left[\mathrm{J}_{\mathrm{P} \mathrm{se}}\right), \mathrm{Hz}\right]\end{array}$ & $\begin{array}{c}\delta_{\mathrm{sec}} \mathrm{ppm} \\
{\left[\left(J_{\mathrm{Pse}}\right), \mathrm{Hz}\right]}\end{array}$ \\
\hline $3 a$ & $c$-Hexyl & $\mathrm{H}$ & $\mathrm{CH}_{2}$ & 79 & $\begin{array}{l}54.9[374 / 791] \\
54.8[371 / 791]\end{array}$ & $\begin{array}{l}398.6[\text { [371], -91.9[7791] } \\
395.5[374],-92.7[791]\end{array}$ \\
\hline 3b & $c$-Hexyl & $\mathrm{H}$ & $\mathrm{CH}_{2} \mathrm{CH}_{2}$ & 80 & $\begin{array}{l}52.5[384 / 789] \\
52.4[386 / 786]\end{array}$ & $\begin{array}{l}378.5[384],-90.1[784] \\
375.6[386],-94.7[786]\end{array}$ \\
\hline $3 \mathrm{c}$ & $c$-Hexyl & $\mathrm{H}$ & $\mathrm{CH}_{2} \mathrm{CH}_{2} \mathrm{CH}_{2}$ & 70 & $\begin{array}{l}53.7[384 / 789] \\
53.6[386 / 786]\end{array}$ & $\begin{array}{l}325.1[384],-102.8[789] \\
324.8[386],-103.5[786]\end{array}$ \\
\hline $3 d$ & $c$-Hexyl & $\mathrm{H}$ & $\begin{array}{c}\mathrm{CH}_{2} \mathrm{CH}_{2} \mathrm{CH}_{2} \mathrm{C} \\
\mathrm{H}_{2}\end{array}$ & 78 & $\begin{array}{l}53.5[384 / 789] \\
53.4[386 / 786]\end{array}$ & $\begin{array}{l}326.7[386],-105.0[789] \\
326.3[388],-105.2[786]\end{array}$ \\
\hline $3 \mathrm{e}$ & $c$-Hexyl & $\mathrm{H}$ & $\begin{array}{c}1,2- \\
\mathrm{CH}_{2} \mathrm{C}_{6} \mathrm{H}_{4} \mathrm{CH}_{2}\end{array}$ & 77 & $\begin{array}{l}53.9[386 / 789] \\
53.8[386 / 789]\end{array}$ & $\begin{array}{l}395.9[386],-96.7[789] \\
395.1[386],-96.8[789]\end{array}$ \\
\hline $3 \mathrm{f}$ & 'Pr & $\mathrm{H}$ & $\mathrm{CH}_{2} \mathrm{CH}_{2}$ & 65 & $\begin{array}{l}53.0[383 / 786] \\
52.7[385 / 784]\end{array}$ & $\begin{array}{l}514.7[385],-248.5[784] \\
514.5[383],-248.7[786]\end{array}$ \\
\hline $3 \mathrm{~g}$ & Pr & $\mathrm{H}$ & $\mathrm{CH}_{2} \mathrm{CH}_{2} \mathrm{CH}_{2}$ & 55 & $\begin{array}{l}53.9[385 / 789] \\
53.8[385 / 789]\end{array}$ & $\begin{array}{l}323.4[385],-164.2[789] \\
323.0[385],-173.8[789]\end{array}$ \\
\hline $3 \mathrm{~h}$ & 'Pr & $\mathrm{H}$ & $\begin{array}{c}1,2-2 \\
\mathrm{CH}_{2} \mathrm{C}_{6} \mathrm{H}_{4} \mathrm{CH}_{2}\end{array}$ & 56 & $\begin{array}{l}54.2[385 / 786] \\
54.1[385 / 786]\end{array}$ & $\begin{array}{l}392.7[385],-99.5[786] \\
392.1[385],-99.7[786]\end{array}$ \\
\hline $3 \mathrm{i}$ & 'Bu & $\mathrm{Bu}$ & $\begin{array}{c}1,2- \\
\mathrm{CH}_{2} \mathrm{C}_{6} \mathrm{H}_{4} \mathrm{CH}_{2}\end{array}$ & 53 & $\begin{array}{l}77.1[386 / 792] \\
76.8[386 / 794] \\
76.2[386 / 794] \\
76.1[386 / 792]\end{array}$ & $\begin{array}{l}357.1 \text { [386], -95.6 [792] } \\
354.1 \text { [386], -96.7 [794] } \\
350.5[386],-98.4[794] \\
350.1[386],-99.1[792]\end{array}$ \\
\hline $3 \mathrm{j}$ & 'Pr & $\mathrm{H}$ & $\begin{array}{c}\text { biphenyl-4,4'- } \\
\text { diylbis(methyle } \\
\text { ne) }\end{array}$ & 54 & $\begin{array}{l}54.0[387 / 789] \\
53.9[387 / 789]\end{array}$ & $\begin{array}{l}408.5[386],-99.1[1789] \\
408.4[385],-99.3[\text { [789] }\end{array}$ \\
\hline $3 \mathbf{k}$ & 'Bu & $\mathrm{H}$ & $\begin{array}{c}1,3- \\
\mathrm{CH}_{2} \mathrm{C}_{6} \mathrm{H}_{4} \mathrm{CH}_{2}\end{array}$ & 59 & $\begin{array}{l}46.1[374 / 794] \\
45.9[367 / 789] \\
45.8[377 / 7899]\end{array}$ & $\begin{array}{l}450.7[374],-49.2[789] \\
447.8[377],-49.8[789] \\
447.2[367],-50.4[789]\end{array}$ \\
\hline 31 & 'Bu & $\mathrm{H}$ & $\begin{array}{c}1,2-2- \\
\mathrm{CH}_{2} \mathrm{C}_{6} \mathrm{H}_{\mathrm{C}} \mathrm{CH}_{2}\end{array}$ & 51 & $\begin{array}{l}46.0[376 / 789] \\
45.9[378 / 786]\end{array}$ & $\begin{array}{l}438.8[378],-50.3[789] \\
438.1[385],-50.4[789]\end{array}$ \\
\hline $3 \mathrm{~m}$ & Bu & $\mathrm{H}$ & $\begin{array}{c}1,2- \\
\mathrm{CH}_{2} \mathrm{C}_{6} \mathrm{H}_{4} \mathrm{CH}_{2}\end{array}$ & 54 & $\begin{array}{l}55.2[384 / 789] \\
55.1[384 / 789] \\
54.8[384 / 789] \\
54.7[387 / 789]\end{array}$ & $\begin{array}{c}395.9[384],-99.0[789] \\
395.2[384],-99.5[789] \\
394.8[384],-101.2[789] \\
391.4[387],-101.4 \text { [789] }\end{array}$ \\
\hline 3n & ${ }^{8} \mathrm{Bu}$ & $\mathrm{H}$ & $1,3-$ & 60 & $55.0[390 / 789]$ & 406.4 [390], - 97.1 [789] \\
\hline
\end{tabular}

\begin{tabular}{|l|l|l|l|l|c|}
\hline & & $\mathrm{CH}_{2} \mathrm{C}_{6} \mathrm{H}_{4} \mathrm{CH}_{2}$ & & $54.9[390 / 789]$ & $406.2[390],-97.5[789]$ \\
& & & & $54.6[390 / 789]$ & $406.1[390],-99.1[789]$ \\
& & & $54.5[390 / 789]$ & $405.7[390],-99.5[789]$ \\
\hline
\end{tabular}

\section{Conclusions}

In conclusion, an efficient route has been developed to prepare a series of new $S e$-alkylphenylphosphonamidodiselenoates and alkane $\quad \operatorname{bis}(N$-alkyl- $P$-phenylphosphonamidodiselenoate)s $s$ in moderate to good yields from the reaction of ammonium phenylphosphonamidodiselenoates

\section{or} phenylphosphonamidodiselenoic diamides, which were derived from WR with primary/secondary amines, with either two molar equivalents of haloalkanes or one molar equivalent of dibromoalkanes. All new compounds were fully elucidated by means of IR, mass spectroscopy and multinuclear NMR in conjunction with single crystal X-ray crystallography of two structures.

\section{Experimental Section}

Unless otherwise stated, all reactions were carried out under on oxygen free nitrogen atmosphere using pre-dried solvents and standard Schlenk techniques, subsequent chromatographic and work up procedures were performed in air. ${ }^{1} \mathrm{H}(270 \mathrm{MHz}),{ }^{13} \mathrm{C}$ (67.9 MHz), ${ }^{31} \mathrm{P}-\left\{{ }^{1} \mathrm{H}\right\} \quad(109 \mathrm{MHz})$ and ${ }^{77} \mathrm{Se}-\left\{{ }^{1} \mathrm{H}\right\} \quad(51.4 \mathrm{MHz}$ referenced to external $\mathrm{Me}_{2} \mathrm{Se}$ ) NMR spectra were recorded at $25^{\circ} \mathrm{C}$ (unless stated otherwise) on a JEOL GSX 270. IR spectra were recorded as $\mathrm{KBr}$ pellets in the range of $4000-250 \mathrm{~cm}^{-1}$ on a PerkinElmer 2000 FTIR/Raman spectrometer. Microanalysis was performed by the University of St-Andrews microanalysis service. Mass spectrometry was performed by the EPSRC National Mass Spectrometry Service Centre, Swansea and the University of St Andrews Mass Spectrometry Service. X-ray crystal data for $\mathbf{2 e}$ and $\mathbf{2 j}$ was collected using a Rigaku SCXMIni Mercury CCD system. Intensity data were collected using $\omega$ steps accumulating area detector images spanning at least a hemisphere of reciprocal space. All data were corrected for Lorentz polarization effects. Absorption effects were corrected on the basis of multiple equivalent reflections or by semi-empirical methods. Structures were solved by direct methods and refined by full-matrix least-squares against $\mathrm{F}^{2}$ by using the program SHELXTL. ${ }^{36}$ Hydrogen atoms were assigned riding isotropic displacement parameters and constrained to idealized geometries. These data can be obtained free of charge via www.ccdc.cam.ac.uk/conts/retrieving.html or from the Cambridge Crystallographic Data centre, 12 Union Road, Cambridge CB2 1EZ, UK; fax (+44) 1223-336-033; e-mail: deposit@ccdc.cam.ac.uk. CCDC 2e 944056; 2 i 944057.

General procedure for synthesis of compounds 2a-n: A mixture of amine (4.0 mmol) and WR (0.54 g, $1.0 \mathrm{mmol})$ in tetrahydrofuran $(60 \mathrm{~mL})$ was stirred at room temperature for $2 \mathrm{~h}$. The brown suspension disappeared and a pale yellow suspension formed. To the mixture, the appropriate haloalkanes $(2.0 \mathrm{mmol})$ was added and the mixture was allowed to stir at room temperature for $24 \mathrm{~h}$. After filtering to remove insoluble solid and drying in vacuo, the residue was dissolved in dichloromethane (ca. $2.0 \mathrm{~mL}$ ) and purified by column chromatography on silica gel using dichloromethane/hexane (1:1) as eluent to give the compounds $\mathbf{2 a -}$ n.

$N$-Cyclohexyl

Se-(2-oxo-2-phenylethyl)

phenylphosphonamidodiselenoate (2a): $0.519 \mathrm{~g}$ as a yellow oil in $54 \%$ yield. Selected IR $\left(\mathrm{KBr}, \mathrm{cm}^{-1}\right): 1670(\mathrm{vs}), 1596(\mathrm{~m}), 1579(\mathrm{~m})$, 1448(s), 1274(vs), 1180(m), 1005(m), 798(m), 748(m), 709(s), 688(s), 605(m). ${ }^{1} \mathrm{H}$ NMR $\left(\mathrm{CD}_{2} \mathrm{Cl}_{2}, \delta\right), 8.06-7.91(\mathrm{~m}, 4 \mathrm{H}, \mathrm{Ar}-\mathrm{H})$, 7.61-7.41 (m, 6H, Ar-H), $4.75\left(\mathrm{~d}, 2 \mathrm{H}, \mathrm{SeCH}_{2}\right), 4.37-4.12(\mathrm{~m}, 1 \mathrm{H}$, cyclohexyl-H), $3.32-3.20(\mathrm{~m}, 1 \mathrm{H}, \mathrm{NH}), 1.96-0.85(\mathrm{~m}, 10 \mathrm{H}$, 
cyclohexyl-H) ppm. ${ }^{13} \mathrm{C}$ NMR $\left(\mathrm{CD}_{2} \mathrm{Cl}_{2}, \delta\right), 194.9(\mathrm{C}=\mathrm{O}), 136.1(\mathrm{~d}$ $J(\mathrm{P}, \mathrm{C})=91.3 \mathrm{~Hz}), 134.0,133.1,132.2(\mathrm{~d}, J(\mathrm{P}, \mathrm{C})=3.1 \mathrm{~Hz}), 130.9$ $(\mathrm{d}, J(\mathrm{P}, \mathrm{C})=12.5 \mathrm{~Hz}), 128.8,128.7,128.5(\mathrm{~d}, J(\mathrm{P}, \mathrm{C})=13.5 \mathrm{~Hz})$, 53.0, 37.5, $35.4(\mathrm{~d}, J(\mathrm{P}, \mathrm{C})=19.7 \mathrm{~Hz}), 35.3(\mathrm{~d}, J(\mathrm{P}, \mathrm{C})=18.7 \mathrm{~Hz})$, $25.1 \mathrm{ppm} .{ }^{31} \mathrm{P}$ NMR $\left(\mathrm{CD}_{2} \mathrm{Cl}_{2}, \delta\right), 53.7(\mathrm{~s}, J(\mathrm{P}, \mathrm{Se})=371 \mathrm{~Hz}, J(\mathrm{P}, \mathrm{Se})$ $=792 \mathrm{~Hz}) \mathrm{ppm} .{ }^{77} \mathrm{Se} \mathrm{NMR}\left(\mathrm{CD}_{2} \mathrm{Cl}_{2}, \delta\right), 345.4(\mathrm{~d}, J(\mathrm{P}, \mathrm{Se})=371$ $\mathrm{Hz}),-102.0(\mathrm{~d}, J(\mathrm{P}, \mathrm{Se})=792 \mathrm{~Hz}) \mathrm{ppm}$. Mass spectrum $\left[\mathrm{CI}^{+}, \mathrm{m} / \mathrm{z}\right]$ $486[\mathrm{M}+\mathrm{H}]^{+}$. Accurate mass measurement $\left(\mathrm{CI}^{+}\right)$: 485.9994, calculate mass for $\mathrm{C}_{20} \mathrm{H}_{24} \mathrm{NOPSe}{ }_{2} \mathrm{H}[\mathrm{M}+\mathrm{H}]^{+}$: 486.0002 .

$N$-Cyclohexyl Se-undecyl phenylphosphonamidodiselenoate (2b): $0.433 \mathrm{~g}$ as a pale yellow oil in $42 \%$ yield. Selected IR (KBr, $\left.\mathrm{cm}^{-1}\right):$ 1446(m), 1436(m), 1403(m), 1292(m), 1231(m), 1136(m), 1081(s), 994(m), 877(m), 745(s), 690(s), 560(s), 524(s). ${ }^{1} \mathrm{H}$ NMR $\left(\mathrm{CD}_{2} \mathrm{Cl}_{2}, \delta\right)$, 8.05-7.97 (m, 2H, Ar-H), 7.49-7.45 (m, 3H, Ar-H), $3.40(\mathrm{dt}, J(\mathrm{H}, \mathrm{H})=7.4 \mathrm{~Hz}, 1 \mathrm{H}$, cyclohexyl-H), 3.22-3.12 $(\mathrm{m}, 2 \mathrm{H}$, cyclohexyl-H), 2.92-2.84 $(\mathrm{m}, J(\mathrm{P}, \mathrm{H})=13.2 \mathrm{~Hz}, J(\mathrm{H}, \mathrm{H})=7.4 \mathrm{~Hz}$, $\left.2 \mathrm{H}, \mathrm{SeCH}_{2}\right), 2.71\left(\mathrm{t}, J(\mathrm{H}, \mathrm{H})=7.4 \mathrm{~Hz}, 2 \mathrm{H}, \mathrm{CH}_{2}\right), 1.96(\mathrm{~d}, J(\mathrm{H}, \mathrm{H})=$ $9.4 \mathrm{~Hz}, 1 \mathrm{H}, \mathrm{NH}), 1.71-1.19\left(\mathrm{~m}, 24 \mathrm{H}\right.$, cyclohexyl-H $\left.+\mathrm{CH}_{2}\right), 0.87$ (t, $\left.J(\mathrm{H}, \mathrm{H})=6.6 \mathrm{~Hz}, 3 \mathrm{H}, \mathrm{CH}_{3}\right) \mathrm{ppm} .{ }^{13} \mathrm{C} \mathrm{NMR}\left(\mathrm{CD}_{2} \mathrm{Cl}_{2}, \delta\right), 137.3(\mathrm{~d}$, $J(\mathrm{P}, \mathrm{C})=87.2 \mathrm{~Hz}), 131.9(\mathrm{~d}, J(\mathrm{P}, \mathrm{C})=3.1 \mathrm{~Hz}), 130.8(\mathrm{~d}, J(\mathrm{P}, \mathrm{C})=$ $12.5 \mathrm{~Hz}), 128.4(\mathrm{~d}, J(\mathrm{P}, \mathrm{C})=13.5 \mathrm{~Hz}), 52.8,35.7(\mathrm{~d}, J(\mathrm{P}, \mathrm{C})=5.2$ $\mathrm{Hz}), 35.6(\mathrm{~d}, J(\mathrm{P}, \mathrm{C})=5.2 \mathrm{~Hz}), 32.9,32.0,30.5(\mathrm{~d}, J(\mathrm{P}, \mathrm{C})=3.1 \mathrm{~Hz})$ 29.9, 29.6, 29.5, 29.4, 29.1, 25.5, 25.2, 22.8, 14.0 ppm. ${ }^{31} \mathrm{P}$ NMR $\left(\mathrm{CD}_{2} \mathrm{Cl}_{2}, \delta\right), 53.2(\mathrm{~s}, J(\mathrm{P}, \mathrm{Se})=391 \mathrm{~Hz}, J(\mathrm{P}, \mathrm{Se})=786 \mathrm{~Hz}) \mathrm{ppm}$. ${ }^{77} \mathrm{Se}$ NMR $\left(\mathrm{CD}_{2} \mathrm{Cl}_{2}, \delta\right), 323.6(\mathrm{~d}, J(\mathrm{P}, \mathrm{Se})=391 \mathrm{~Hz}),-104.5(\mathrm{~d}$, $J(\mathrm{P}, \mathrm{Se})=786 \mathrm{~Hz}) \mathrm{ppm}$. Mass spectrum $\left[\mathrm{CI}^{+}, \mathrm{m} / \mathrm{z}\right]: 522[\mathrm{M}+\mathrm{H}]^{+}$. Accurate mass measurement $\left(\mathrm{CI}^{+}\right): 522.1299$, calculate mass for $\mathrm{C}_{23} \mathrm{H}_{40} \mathrm{NPSe}{ }_{2} \mathrm{H}[\mathrm{M}+\mathrm{H}]^{+}: 522.1303$.

$\mathrm{N}$-Cyclohexyl $\mathrm{Se}$-nonyl phenylphosphonamidodiselenoate (2c): $0.405 \mathrm{~g}$ as a colourless oil in $41 \%$ yield. Selected IR $\left(\mathrm{KBr}, \mathrm{cm}^{-1}\right)$ : 1447(m), 1436(m), 1404(m), 1293(m), 1231(m), 1142(m), 1081(s), 994(m), 878(m), 746(s), 690(s), 569(s), 524(s). ${ }^{1} \mathrm{H}$ NMR $\left(\mathrm{CD}_{2} \mathrm{Cl}_{2}\right.$,

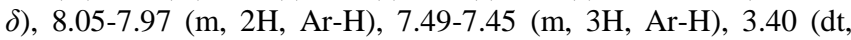
$J(\mathrm{H}, \mathrm{H})=7.4 \mathrm{~Hz}, 1 \mathrm{H}$, cyclohexyl-H $), 3.22-3.12(\mathrm{~m}, 2 \mathrm{H}$, cyclohexyl-H), 2.92-2.84 (m, $J(\mathrm{P}, \mathrm{H})=13.2 \mathrm{~Hz}, J(\mathrm{H}, \mathrm{H})=7.4 \mathrm{~Hz}$, $\left.2 \mathrm{H}, \mathrm{SeCH}_{2}\right), 2.72\left(\mathrm{t}, J(\mathrm{H}, \mathrm{H})=7.4 \mathrm{~Hz}, 2 \mathrm{H}, \mathrm{CH}_{2}\right), 1.96(\mathrm{~d}, J(\mathrm{H}, \mathrm{H})=$ $9.4 \mathrm{~Hz}, 1 \mathrm{H}, \mathrm{NH}), 1.71-1.18\left(\mathrm{~m}, 20 \mathrm{H}\right.$, cyclohexyl-H $\left.+\mathrm{CH}_{2}\right), 0.87(\mathrm{t}$, $\left.J(\mathrm{H}, \mathrm{H})=6.6 \mathrm{~Hz}, 3 \mathrm{H}, \mathrm{CH}_{3}\right) \mathrm{ppm} .{ }^{13} \mathrm{C} \mathrm{NMR}\left(\mathrm{CD}_{2} \mathrm{Cl}_{2}, \delta\right), 137.3(\mathrm{~d}$, $J(\mathrm{P}, \mathrm{C})=86.2 \mathrm{~Hz}), 131.8(\mathrm{~d}, J(\mathrm{P}, \mathrm{C})=3.1 \mathrm{~Hz}), 130.8(\mathrm{~d}, J(\mathrm{P}, \mathrm{C})=$ $12.5 \mathrm{~Hz}), 128.3(\mathrm{~d}, J(\mathrm{P}, \mathrm{C})=13.5 \mathrm{~Hz}), 52.8,35.6(\mathrm{~d}, J(\mathrm{P}, \mathrm{C})=5.2$ $\mathrm{Hz}), 35.5(\mathrm{~d}, J(\mathrm{P}, \mathrm{C})=5.2 \mathrm{~Hz}), 32.9,31.9,30.5(\mathrm{~d}, J(\mathrm{P}, \mathrm{C})=3.1 \mathrm{~Hz})$ 29.9, 29.5, 29.4, 29.3, 25.5, 25.2, 22.8, 14.0 ppm. ${ }^{31} \mathrm{P}$ NMR $\left(\mathrm{CD}_{2} \mathrm{Cl}_{2}, \delta\right), 53.2(\mathrm{~s}, J(\mathrm{P}, \mathrm{Se})=390 \mathrm{~Hz}, J(\mathrm{P}, \mathrm{Se})=786 \mathrm{~Hz}) \mathrm{ppm}$. ${ }^{77} \mathrm{Se} \mathrm{NMR}\left(\mathrm{CD}_{2} \mathrm{Cl}_{2}, \delta\right), 323.6(\mathrm{~d}, J(\mathrm{P}, \mathrm{Se})=390 \mathrm{~Hz}),-104.6(\mathrm{~d}$, $J(\mathrm{P}, \mathrm{Se})=786 \mathrm{~Hz}) \mathrm{ppm}$. Mass spectrum $\left[\mathrm{CI}^{+}, \mathrm{m} / \mathrm{z}\right]: 494[\mathrm{M}+\mathrm{H}]^{+}$. Accurate mass measurement $\left(\mathrm{CI}^{+}\right)$: 494.0892, calculate mass for $\mathrm{C}_{21} \mathrm{H}_{36} \mathrm{NPSe}_{2} \mathrm{H}[\mathrm{M}+\mathrm{H}]^{+}: 494.0990$.

$N$-Cyclohexyl $S e$-heptyl phenylphosphonamidodiselenoate (2d): $0.272 \mathrm{~g}$ as a colourless oil in $59 \%$ yield. Selected $\mathrm{IR}\left(\mathrm{KBr}, \mathrm{cm}^{-1}\right)$ : $1450(\mathrm{~m}), \quad 1436(\mathrm{~m}), \quad 1404(\mathrm{~m}), \quad 1293(\mathrm{~m}), \quad 1231(\mathrm{~m}), \quad 1140(\mathrm{~m})$, 1081(vs), 994(m), 878(m), 746(s), 690(s), 570(s), 523(s). ${ }^{1} \mathrm{H}$ NMR $\left(\mathrm{CD}_{2} \mathrm{Cl}_{2}, \delta\right), 8.06-7.97(\mathrm{~m}, 2 \mathrm{H}, \mathrm{Ar}-\mathrm{H}), 7.50-7.45(\mathrm{~m}, 3 \mathrm{H}, \mathrm{Ar}-\mathrm{H})$, 3.17-3.12 $(\mathrm{m}, 1 \mathrm{H}$, cyclohexyl-H), $2.89(\mathrm{dt}, J(\mathrm{P}, \mathrm{H})=13.2 \mathrm{~Hz}$, $\left.J(\mathrm{H}, \mathrm{H})=7.4 \mathrm{~Hz}, 2 \mathrm{H}, \mathrm{SeCH}_{2}\right), 2.72\left(\mathrm{t}, J(\mathrm{H}, \mathrm{H})=7.4 \mathrm{~Hz}, 2 \mathrm{H}, \mathrm{CH}_{2}\right)$, $1.96(\mathrm{~d}, J(\mathrm{H}, \mathrm{H})=9.4 \mathrm{~Hz}, 1 \mathrm{H}, \mathrm{NH}), 1.69-1.09(\mathrm{~m}, 18 \mathrm{H}$, cyclohexyl$\left.\mathrm{H}+\mathrm{CH}_{2}\right), 0.86\left(\mathrm{t}, J(\mathrm{H}, \mathrm{H})=6.6 \mathrm{~Hz}, 3 \mathrm{H}, \mathrm{CH}_{3}\right) \mathrm{ppm} .{ }^{13} \mathrm{C} \mathrm{NMR}$ $\left(\mathrm{CD}_{2} \mathrm{Cl}_{2}, \delta\right), 137.2(\mathrm{~d}, J(\mathrm{P}, \mathrm{C})=87.2 \mathrm{~Hz}), 131.9(\mathrm{~d}, J(\mathrm{P}, \mathrm{C})=3.1$ $\mathrm{Hz}), 130.8(\mathrm{~d}, J(\mathrm{P}, \mathrm{C})=12.5 \mathrm{~Hz}), 128.4(\mathrm{~d}, J(\mathrm{P}, \mathrm{C})=13.5 \mathrm{~Hz}), 52.9$, $35.7(\mathrm{~d}, J(\mathrm{P}, \mathrm{C})=5.2 \mathrm{~Hz}), 35.6(\mathrm{~d}, J(\mathrm{P}, \mathrm{C})=5.2 \mathrm{~Hz}), 32.9,31.7$, $30.5(\mathrm{~d}, J(\mathrm{P}, \mathrm{C})=3.1 \mathrm{~Hz}), 29.9,28.8,25.3,22.7,13.9 \mathrm{ppm} .{ }^{31} \mathrm{P}$ $\operatorname{NMR}\left(\mathrm{CD}_{2} \mathrm{Cl}_{2}, \delta\right), 53.2(\mathrm{~s}, J(\mathrm{P}, \mathrm{Se})=390 \mathrm{~Hz}, J(\mathrm{P}, \mathrm{Se})=786 \mathrm{~Hz})$ ppm. ${ }^{77} \mathrm{Se} \mathrm{NMR}\left(\mathrm{CD}_{2} \mathrm{Cl}_{2}, \delta\right), 323.6(\mathrm{~d}, J(\mathrm{P}, \mathrm{Se})=391 \mathrm{~Hz}),-104.4(\mathrm{~d}$ $J(\mathrm{P}, \mathrm{Se})=786 \mathrm{~Hz}) \mathrm{ppm}$. Mass spectrum $\left[\mathrm{ES}^{+}, \mathrm{m} / \mathrm{z}\right]: 488[\mathrm{M}+\mathrm{Na}]^{+}$. Accurate mass measurement $\left(\mathrm{ES}^{+}\right): 488.0500$, calculate mass for $\mathrm{C}_{19} \mathrm{H}_{32} \mathrm{NNaPSe}_{2}[\mathrm{M}+\mathrm{Na}]^{+}: 488.0500$.
$N$-tert-Butyl

Se-[2-(4-bromophenyl)-2-oxoethyl] phenylphosphinodiselenoate (2e): $0.440 \mathrm{~g}$ as a pale yellow paste in $82 \%$ yield. Selected IR $\left(\mathrm{KBr}, \mathrm{cm}^{-1}\right): 1669(\mathrm{~s}), 1582(\mathrm{~s}), 1564(\mathrm{~m})$, 1474(m), 1437(m), 1385(m), 1363(m), 1270(m), 1222(m), 1188(s), 1095(m), 1070(m), 983(s), 804(m), 747(s), 691(s), 546(m), 528(s). ${ }^{1} \mathrm{H}$ NMR $\left(\mathrm{CD}_{2} \mathrm{Cl}_{2}, \delta\right), 8.12-8.04(\mathrm{~m}, 2 \mathrm{H}, \mathrm{Ar}-\mathrm{H}), 7.84(\mathrm{~d}, J(\mathrm{H}, \mathrm{H})=$ $8.8 \mathrm{~Hz}, 2 \mathrm{H}, \mathrm{Ar}-\mathrm{H}), 7.60(\mathrm{~d}, J(\mathrm{H}, \mathrm{H})=8.8 \mathrm{~Hz}, 2 \mathrm{H}, \mathrm{Ar}-\mathrm{H}), 7.51-7.43$ $(\mathrm{m}, 3 \mathrm{H}, \mathrm{Ar}-\mathrm{H}), 4.37\left(\mathrm{~d}, J(\mathrm{P}, \mathrm{H})=13.2 \mathrm{~Hz}, 2 \mathrm{H}, \mathrm{SeCH}_{2}\right), 2.54(\mathrm{~d}$, $J(\mathrm{H}, \mathrm{H})=9.5 \mathrm{~Hz}, 1 \mathrm{H}, \mathrm{NH}), 1.30\left(\mathrm{~s}, 9 \mathrm{H}, \mathrm{CH}_{3}\right) \mathrm{ppm} .{ }^{13} \mathrm{C} \mathrm{NMR}$ $\left(\mathrm{CD}_{2} \mathrm{Cl}_{2}, \delta\right), 194.3(\mathrm{C}=\mathrm{O}), 137.7(\mathrm{~d}, J(\mathrm{P}, \mathrm{C})=91.3 \mathrm{~Hz}), 134.4$, $132.1,131.2,131.0,130.4,128.6,128.4,58.0,38.5,31.1 \mathrm{ppm} .{ }^{31} \mathrm{P}$ $\operatorname{NMR}\left(\mathrm{CD}_{2} \mathrm{Cl}_{2}, \delta\right), 46.9(\mathrm{~s}, J(\mathrm{P}, \mathrm{Se})=355 \mathrm{~Hz}, J(\mathrm{P}, \mathrm{Se})=794 \mathrm{~Hz})$ ppm. ${ }^{77} \mathrm{Se} \mathrm{NMR}\left(\mathrm{CD}_{2} \mathrm{Cl}_{2}, \delta\right), 384.0(\mathrm{~d}, J(\mathrm{P}, \mathrm{Se})=355 \mathrm{~Hz}),-53.0(\mathrm{~d}$, $J(\mathrm{P}, \mathrm{Se})=792 \mathrm{~Hz}) \mathrm{ppm}$. Mass spectrum $\left[\mathrm{ES}^{+}, \mathrm{m} / \mathrm{z}\right]: 560[\mathrm{M}+\mathrm{Na}]^{+}$. Accurate mass measurement $\left(\mathrm{ES}^{+}\right)$: 559.8767, calculate mass for $\mathrm{C}_{18} \mathrm{H}_{21} \mathrm{BrNONaPSe}_{2}[\mathrm{M}+\mathrm{Na}]^{+}: 559.8772$.

\section{$N$-sec-Butyl}

Se-[2-(4-bromophenyl)-2-oxoethyl] phenylphosphonamidodiselenoate (2f): $0.500 \mathrm{~g}$ as a reddish yellow sticky oil in $93 \%$ yield. Two stereoisomers were found in ca. $1: 1$ intensity ratio. Selected IR $\left(\mathrm{KBr}, \mathrm{cm}^{-1}\right): 1671(\mathrm{~s}), 1583(\mathrm{~s})$, 1435(m), 1397(s), 1273(s), 1178(s), 1098(m), 1070(s), 1004(s), 838(m), 746(s), 689(m), 538(s). ${ }^{1} \mathrm{H}$ NMR $\left(\mathrm{CD}_{2} \mathrm{Cl}_{2}, \delta\right), 8.04-7.94$ (m, 2x2H, Ar-H), 7.81-7.75 (m, 2x2H, Ar-H), 7.61-7.54 (m, 2x2H, Ar-H), 7.49-7.45 (m, 3H, Ar-H), 4.27-3.91 (m, 2x1H, CH), 3.51$3.05\left(\mathrm{~m}, 2 \times 2 \mathrm{H}, \mathrm{SeCH}_{2}\right), 2.54(\mathrm{~d}, J(\mathrm{H}, \mathrm{H})=9.5 \mathrm{~Hz}, 2 \times 1 \mathrm{H}, \mathrm{NH})$, $1.59-1.41\left(\mathrm{~m}, 2 \times 2 \mathrm{H}, \mathrm{CH}_{2}\right), 1.13(\mathrm{dd}, J(\mathrm{H}, \mathrm{H})=6.6 \mathrm{~Hz}, J(\mathrm{P}, \mathrm{H})=3.3$ $\left.\mathrm{Hz}, 3 \mathrm{H}, \mathrm{CH}_{3}\right), 1.12\left(\mathrm{dd}, J(\mathrm{H}, \mathrm{H})=6.6 \mathrm{~Hz}, J(\mathrm{P}, \mathrm{H})=3.3 \mathrm{~Hz}, 3 \mathrm{H}, \mathrm{CH}_{3}\right)$, $0.88\left(\mathrm{t}, J(\mathrm{H}, \mathrm{H})=7.4 \mathrm{~Hz}, 3 \mathrm{H}, \mathrm{CH}_{3}\right), 0.85(\mathrm{t}, J(\mathrm{H}, \mathrm{H})=7.4 \mathrm{~Hz}, 3 \mathrm{H}$, $\left.\mathrm{CH}_{3}\right) \mathrm{ppm} .{ }^{13} \mathrm{C} \mathrm{NMR}\left(\mathrm{CD}_{2} \mathrm{Cl}_{2}, \delta\right), 194.4(\mathrm{C}=\mathrm{O}), 136.3(\mathrm{~d}, J(\mathrm{P}, \mathrm{C})=$ $89.4 \mathrm{~Hz}), 136.2(\mathrm{~d}, J(\mathrm{P}, \mathrm{C})=90.6 \mathrm{~Hz}), 134.6,134.5,132.6(\mathrm{~d}$, $J(\mathrm{P}, \mathrm{C})=3.1 \mathrm{~Hz}), 132.5(\mathrm{~d}, J(\mathrm{P}, \mathrm{C})=3.1 \mathrm{~Hz}), 132.4,132.3,132.2$, 131.5, 131.4, 131.3, 131.2, 130.7, 130.6, 129.1, 129.0, 128.3, 128.7, $51.7(\mathrm{~d}, J(\mathrm{P}, \mathrm{C})=18.9 \mathrm{~Hz}), 51.4(\mathrm{~d}, J(\mathrm{P}, \mathrm{C})=18.7 \mathrm{~Hz}), 38.1,37.5$, $31.8(\mathrm{~d}, J(\mathrm{P}, \mathrm{C})=23.6 \mathrm{~Hz}), 31.7(\mathrm{~d}, J(\mathrm{P}, \mathrm{C})=22.1 \mathrm{~Hz}), 22.2(\mathrm{~d}$, $J(\mathrm{P}, \mathrm{C})=21.4 \mathrm{~Hz}), 22.1(\mathrm{~d}, J(\mathrm{P}, \mathrm{C})=20.5 \mathrm{~Hz}), 10.5,10.4 \mathrm{ppm} .{ }^{31} \mathrm{P}$ NMR $\left(\mathrm{CD}_{2} \mathrm{Cl}_{2}, \delta\right), 56.0(\mathrm{~s}, J(\mathrm{P}, \mathrm{Se})=366 \mathrm{~Hz}, J(\mathrm{P}, \mathrm{Se})=796 \mathrm{~Hz})$, $54.8(\mathrm{~s}, J(\mathrm{P}, \mathrm{Se})=373 \mathrm{~Hz}, J(\mathrm{P}, \mathrm{Se})=782 \mathrm{~Hz}) \mathrm{ppm} .{ }^{77} \mathrm{Se} \mathrm{NMR}$ $\left(\mathrm{CD}_{2} \mathrm{Cl}_{2}, \delta\right), 348.5(\mathrm{~d}, J(\mathrm{P}, \mathrm{Se})=374 \mathrm{~Hz}), 346.1(\mathrm{~d}, J(\mathrm{P}, \mathrm{Se})=367$ $\mathrm{Hz}),-101.9(\mathrm{~d}, J(\mathrm{P}, \mathrm{Se})=794 \mathrm{~Hz}),-104.8(\mathrm{~d}, J(\mathrm{P}, \mathrm{Se})=784 \mathrm{~Hz})$ ppm. Mass spectrum $\left[\mathrm{ES}^{+}, \mathrm{m} / \mathrm{z}\right]: 560[\mathrm{M}+\mathrm{Na}]^{+}$. Accurate mass measurement $\left(\mathrm{ES}^{+}\right)$: 559.8762, calculate mass for $\mathrm{C}_{18} \mathrm{H}_{21} \mathrm{BrNONaPSe}_{2}[\mathrm{M}+\mathrm{Na}]^{+}: 559.8772$.

\section{$N$-Butyl Se-[2-(4-bromophenyl)-2-oxoethyl]} phenylphosphonamidodiselenoate (2g): $0.434 \mathrm{~g}$ as a yellow sticky oil in $81 \%$ yield. Selected IR $\left(\mathrm{KBr}, \mathrm{cm}^{-1}\right): 1657(\mathrm{~s}), 1585(\mathrm{~s})$, 1435(m), 1396(m), 1279(s), 1100(m), 1085(m), 1069(m), 1004(s), 841(m), 752(s), 705(m), 689(m), 567(s), 441(m). ${ }^{1} \mathrm{H}$ NMR $\left(\mathrm{CD}_{2} \mathrm{Cl}_{2}\right.$, $\delta), 8.00-7.92(\mathrm{~m}, 2 \mathrm{H}, \mathrm{Ar}-\mathrm{H}), 7.81(\mathrm{~d}, J(\mathrm{H}, \mathrm{H})=6.9 \mathrm{~Hz}, 2 \mathrm{H}, \mathrm{Ar}-\mathrm{H})$, $7.61(\mathrm{~d}, J(\mathrm{H}, \mathrm{H})=6.9 \mathrm{~Hz}, 2 \mathrm{H}, \mathrm{Ar}-\mathrm{H}), 7.50-7.47(\mathrm{~m}, 3 \mathrm{H}, \mathrm{Ar}-\mathrm{H})$, 4.31-4.15 (m, 2H, $\left.\mathrm{NHCH}_{2}\right), 3.18$ (ws, $\left.1 \mathrm{H}, \mathrm{NH}\right), 2.91(\mathrm{~d}, 2 \mathrm{H}$, $\left.\mathrm{SeCH}_{2}\right), 1.62-1.28(\mathrm{~m}, 4 \mathrm{H}$, cyclohexyl-H), $0.87(\mathrm{t}, J(\mathrm{H}, \mathrm{H})=7.4 \mathrm{~Hz}$, $\left.3 \mathrm{H}, \mathrm{CH}_{3}\right)$ ppm. ${ }^{13} \mathrm{C}$ NMR $\left(\mathrm{CD}_{2} \mathrm{Cl}_{2}, \delta\right), 194.4(\mathrm{C}=\mathrm{O}), 135.4(\mathrm{~d}$, $J(\mathrm{P}, \mathrm{C})=89.9 \mathrm{~Hz}), 134.5,132.8(\mathrm{~d}, J(\mathrm{P}, \mathrm{C})=3.1 \mathrm{~Hz}), 132.4,131.1$ $(\mathrm{d}, J(\mathrm{P}, \mathrm{C})=12.5 \mathrm{~Hz}), 130.7,129.1(\mathrm{~d}, J(\mathrm{P}, \mathrm{C})=13.5 \mathrm{~Hz}), 43.5(\mathrm{~d}$, $J(\mathrm{P}, \mathrm{C})=3.7 \mathrm{~Hz}), 37.2,33.3(\mathrm{~d}, J(\mathrm{P}, \mathrm{C})=9.5 \mathrm{~Hz}), 20.4,13.9 \mathrm{ppm}$. ${ }^{31} \mathrm{P}$ NMR $\left(\mathrm{CD}_{2} \mathrm{Cl}_{2}, \delta\right), 58.0(\mathrm{~s}, J(\mathrm{P}, \mathrm{Se})=370 \mathrm{~Hz}, J(\mathrm{P}, \mathrm{Se})=794 \mathrm{~Hz})$ ppm. ${ }^{77} \mathrm{Se} \mathrm{NMR}\left(\mathrm{CD}_{2} \mathrm{Cl}_{2}, \delta\right), 321.2(\mathrm{~d}, J(\mathrm{P}, \mathrm{Se})=370 \mathrm{~Hz}),-100.4(\mathrm{~d}$, $J(\mathrm{P}, \mathrm{Se})=794 \mathrm{~Hz})$, ppm. Mass spectrum $\left[\mathrm{ES}^{+}, \mathrm{m} / \mathrm{z}\right]: 560[\mathrm{M}+\mathrm{Na}]^{+}$. Accurate mass measurement $\left(\mathrm{ES}^{+}\right)$: 559.8771, calculate mass for $\mathrm{C}_{18} \mathrm{H}_{21} \mathrm{NONaBrPSe}_{2}[\mathrm{M}+\mathrm{Na}]^{+}: 559.8772$.

$\mathrm{N}$-Cyclohexyl Se-[2-(4-bromophenyl)-2-oxoethyl] phenylphosphonamidodiselenoate (2h): $0.630 \mathrm{~g}$ as a brown paste in 56\% yield. Selected IR (KBr, cm $\left.{ }^{-1}\right): 1673(\mathrm{~s}), 1584(\mathrm{~s}), 1483(\mathrm{~m})$, 1435(m), 1396(m), 1270(s), 1178(m), 1071(s), 1004(s), 836(m), 747(s), 689(m), 519(s). ${ }^{1} \mathrm{H}$ NMR $\left(\mathrm{CD}_{2} \mathrm{Cl}_{2}, \delta\right), 8.02-7.97(\mathrm{~m}, 2 \mathrm{H}$, Ar-H), 7.80-7.78 (m, 2H, Ar-H), 7.62-7.58 (m, 2H, Ar-H), 7.507.43 (m, 3H, Ar-H), 4.30-4.14 (m, 1H, cyclohexyl-H), 3.23 (ws, 
$1 \mathrm{H}, \mathrm{NH}), \quad 2.59-2.52\left(\mathrm{~d}, 2 \mathrm{H}, \quad \mathrm{SeCH}_{2}\right), 2.05-0.86(\mathrm{~m}, \quad 10 \mathrm{H}$, cyclohexyl-H) ppm. ${ }^{13} \mathrm{C}$ NMR $\left(\mathrm{CD}_{2} \mathrm{Cl}_{2}, \delta\right), 194.3(\mathrm{C}=\mathrm{O}), 136.3(\mathrm{~d}$, $J(\mathrm{P}, \mathrm{C})=90.0 \mathrm{~Hz}), 134.5,132.6(\mathrm{~d}, J(\mathrm{P}, \mathrm{C})=3.1 \mathrm{~Hz}), 132.4,131.1$ $(\mathrm{d}, J(\mathrm{P}, \mathrm{C})=12.5 \mathrm{~Hz}), 130.7,129.1(\mathrm{~d}, J(\mathrm{P}, \mathrm{C})=13.5 \mathrm{~Hz}), 53.1(\mathrm{~d}$ $J(\mathrm{P}, \mathrm{C})=18.1 \mathrm{~Hz}), 37.8,35.8,29.0,25.3$ ppm. ${ }^{31} \mathrm{P} \mathrm{NMR}\left(\mathrm{CD}_{2} \mathrm{Cl}_{2}\right.$, $\delta), 54.5(\mathrm{~s}, J(\mathrm{P}, \mathrm{Se})=369 \mathrm{~Hz}, J(\mathrm{P}, \mathrm{Se})=794 \mathrm{~Hz}) \mathrm{ppm} .{ }^{77} \mathrm{Se} \mathrm{NMR}$ $\left(\mathrm{CD}_{2} \mathrm{Cl}_{2}, \delta\right), 347.7(\mathrm{~d}, J(\mathrm{P}, \mathrm{Se})=369 \mathrm{~Hz}),-100.7(\mathrm{~d}, J(\mathrm{P}, \mathrm{Se})=794$ $\mathrm{Hz})$ ppm. Mass spectrum $\left[\mathrm{CI}^{+}, \mathrm{m} / \mathrm{z}\right]: 564[\mathrm{M}+\mathrm{H}]^{+}$. Accurate mass measurement $\left(\mathrm{CI}^{+}\right)$: 563.9111, calculate mass for $\mathrm{C}_{20} \mathrm{H}_{23} \mathrm{BrNOPSe}_{2} \mathrm{H}[\mathrm{M}+\mathrm{H}]^{+}:$: 563.9109 .

$N$-Cyclopentyl Se-[2-(4-bromophenyl)-2-oxoethyl] phenylphosphonamidodiselenoate (2i): $0.445 \mathrm{~g}$ as a greyish yellow paste in $81 \%$ yield. Selected IR $\left(\mathrm{KBr}, \mathrm{cm}^{-1}\right)$ : $1658(\mathrm{~s}) \mathrm{m}$, 1581(s), 1434(m), 1416(m), 1395(m), 1278(s), 1177(m), 1093(m), 1068(m), 1004(s), 840(s), 752(s), 704(m), 687(m), 621(m), 555(s), 463(s), 418(m). ${ }^{1} \mathrm{H}$ NMR $\left(\mathrm{CD}_{2} \mathrm{Cl}_{2}, \delta\right), 8.02-7.93$ (m, 2H, Ar-H), $7.81(\mathrm{~d}, J(\mathrm{H}, \mathrm{H})=6.9 \mathrm{~Hz}, 2 \mathrm{H}, \mathrm{Ar}-\mathrm{H}), 7.58(\mathrm{~d}, J(\mathrm{H}, \mathrm{H})=6.9 \mathrm{~Hz}, 2 \mathrm{H}$, Ar-H), 7.52-7.42 (m, 3H, Ar-H), 4.32-4.12 (m, 1H, cyclopentyl-H), $3.31(\mathrm{dd}, 1 \mathrm{H}, \mathrm{NH}), 2.54\left(\mathrm{~d}, 2 \mathrm{H}, \mathrm{SeCH}_{2}\right), 1.94-1.45(\mathrm{~m}, 8 \mathrm{H}$, cyclopentyl-H) ppm. ${ }^{13} \mathrm{C}$ NMR $\left(\mathrm{CD}_{2} \mathrm{Cl}_{2}, \delta\right), 194.1(\mathrm{~d}, J(\mathrm{P}, \mathrm{C})=3.4$ $\mathrm{Hz}, \mathrm{C}=\mathrm{O}), 135.6(\mathrm{~d}, J(\mathrm{P}, \mathrm{C})=90.3 \mathrm{~Hz}), 134.2,132.4(\mathrm{~d}, J(\mathrm{P}, \mathrm{C})=$ $3.1 \mathrm{~Hz}), 132.0,130.9(\mathrm{~d}, J(\mathrm{P}, \mathrm{C})=12.5 \mathrm{~Hz}), 130.4,128.7(\mathrm{~d}, J(\mathrm{P}, \mathrm{C})$ $=13.5 \mathrm{~Hz}), 55.8(\mathrm{~d}, J(\mathrm{P}, \mathrm{C})=4.2 \mathrm{~Hz}), 37.2,34.8(\mathrm{~d}, J(\mathrm{P}, \mathrm{C})=6.2$ $\mathrm{Hz}), 23.3(\mathrm{~d}, J(\mathrm{P}, \mathrm{C})=7.3 \mathrm{~Hz}) \mathrm{ppm} .{ }^{31} \mathrm{P} \mathrm{NMR}\left(\mathrm{CD}_{2} \mathrm{Cl}_{2}, \delta\right), 55.8(\mathrm{~s}$, $J(\mathrm{P}, \mathrm{Se})=369 \mathrm{~Hz}, J(\mathrm{P}, \mathrm{Se})=791 \mathrm{~Hz}) \mathrm{ppm} .{ }^{77} \mathrm{Se} \mathrm{NMR}\left(\mathrm{CD}_{2} \mathrm{Cl}_{2}, \delta\right)$, $338.8(\mathrm{~d}, J(\mathrm{P}, \mathrm{Se})=369 \mathrm{~Hz}),-95.3(\mathrm{~d}, J(\mathrm{P}, \mathrm{Se})=791 \mathrm{~Hz}) \mathrm{ppm}$. Mass spectrum $\left[\mathrm{ES}^{+}, \mathrm{m} / \mathrm{z}\right]: 572[\mathrm{M}+\mathrm{Na}]^{+}$. Accurate mass measurement $\left(\mathrm{ES}^{+}\right)$: 571.8766, calculate mass for $\mathrm{C}_{19} \mathrm{H}_{21} \mathrm{NONaBrPSe}_{2}[\mathrm{M}+\mathrm{Na}]^{+}: 571.8772$.

\section{$N$-Isopropyl}

Se-[2-(4-bromophenyl)-2-oxoethyl] phenylphosphonamidodiselenoate (2j): $0.460 \mathrm{~g}$ as a light yellow paste in $88 \%$ yield. Selected IR $\left(\mathrm{KBr}, \mathrm{cm}^{-1}\right): 1656(\mathrm{~s}), 1580(\mathrm{~s})$, 1563(m), 1413(s), 1277(s), 1124(m), 1094(m), 1068(m), 1029(m), 1004(s), 748(s), 703(m), 687(m), 622(m), 565(s), 473(m), 585(m), $420(\mathrm{~m}) .{ }^{1} \mathrm{H}$ NMR $\left(\mathrm{CD}_{2} \mathrm{Cl}_{2}, \delta\right), 8.01(\mathrm{dd}, J(\mathrm{P}, \mathrm{H})=14.9 \mathrm{~Hz}, J(\mathrm{H}, \mathrm{H})$ $=8.0 \mathrm{~Hz}, 2 \mathrm{H}, \mathrm{Ar}-\mathrm{H}), 7.80(\mathrm{~d}, J(\mathrm{H}, \mathrm{H})=8.5 \mathrm{~Hz}, 2 \mathrm{H}, \mathrm{Ar}-\mathrm{H}), 7.60(\mathrm{~d}$, $J(\mathrm{H}, \mathrm{H})=8.5 \mathrm{~Hz}, 2 \mathrm{H}, \mathrm{Ar}-\mathrm{H}), 7.52-7.43(\mathrm{~m}, 3 \mathrm{H}, \mathrm{Ar}-\mathrm{H}), 4.31-4.08$ $(\mathrm{m}, 1 \mathrm{H}, \mathrm{CH}), 3.65-3.52\left(\mathrm{~m}, 2 \mathrm{H}, \mathrm{SeCH}_{2}\right), 3.19(\mathrm{w}, 1 \mathrm{H}, \mathrm{NH}), 1.15(\mathrm{~d}$ $\left.\mathrm{J}(\mathrm{H}, \mathrm{H})=6.1 \mathrm{~Hz}, 6 \mathrm{H}, \mathrm{CH}_{3}\right) \mathrm{ppm} .{ }^{13} \mathrm{C} \mathrm{NMR}\left(\mathrm{CD}_{2} \mathrm{Cl}_{2}, \delta\right), 194.0$ $(\mathrm{C}=\mathrm{O}), 135.8(\mathrm{~d}, J(\mathrm{P}, \mathrm{C})=90.3 \mathrm{~Hz}, \mathrm{Ar}-\mathrm{C}), 134.2(\mathrm{Ar}-\mathrm{C}), 132.3(\mathrm{~d}$, $J(\mathrm{P}, \mathrm{C})=3.1 \mathrm{~Hz}, \mathrm{Ar}-\mathrm{C})), 132.1(\mathrm{Ar}-\mathrm{C}), 130.9(\mathrm{~d}, J(\mathrm{P}, \mathrm{C})=12.5 \mathrm{~Hz}$, Ar-C) ), 130.4 (Ar-C), $128.6(\mathrm{~d}, J(\mathrm{P}, \mathrm{C})=13.5 \mathrm{~Hz}, \mathrm{Ar}-\mathrm{C})), 46.2(\mathrm{~N}-$ C), $37.4(\mathrm{Se}-\mathrm{C}), 25.0\left(\mathrm{~d}, J(\mathrm{P}, \mathrm{C})=5.2 \mathrm{~Hz}, \mathrm{CH}_{3}\right), 24.6(\mathrm{~d}, J(\mathrm{P}, \mathrm{C})=$ $\left.5.2 \mathrm{~Hz}, \mathrm{CH}_{3}\right) \mathrm{ppm} .{ }^{31} \mathrm{P} \mathrm{NMR}\left(\mathrm{CD}_{2} \mathrm{Cl}_{2}, \delta\right), 54.6(\mathrm{~s}, J(\mathrm{P}, \mathrm{Se})=369 \mathrm{~Hz}$ $J(\mathrm{P}, \mathrm{Se})=794 \mathrm{~Hz}) \mathrm{ppm} .{ }^{77} \mathrm{Se} \mathrm{NMR}\left(\mathrm{CD}_{2} \mathrm{Cl}_{2}, \delta\right), 345.3(\mathrm{~d}, J(\mathrm{P}, \mathrm{Se})=$ $370 \mathrm{~Hz}),-102.4(\mathrm{~d}, J(\mathrm{P}, \mathrm{Se})=794 \mathrm{~Hz}) \mathrm{ppm}$. Mass spectrum $\left[\mathrm{CI}^{+}\right.$ $\mathrm{m} / \mathrm{z}]: 524[\mathrm{M}+\mathrm{H}]^{+}$. Accurate mass measurement $\left(\mathrm{CI}^{+}\right): 523.8791$, calculate mass for $\mathrm{C}_{17} \mathrm{H}_{19} \mathrm{BrNOPSe}_{2} \mathrm{H}[\mathrm{M}+\mathrm{H}]^{+}: 523.8789$.

\section{$N, N$-Diisopropyl $\quad S e$-[2-(4-bromophenyl)-2-oxoethyl} phenylphosphonamidodiselenoate (2k): $0.310 \mathrm{~g}$ as a yellow paste in 55\% yield. Selected IR (KBr, $\left.\mathrm{cm}^{-1}\right)$ : 1682(s), 1585(s), 1434(m), 1394(s), 1362(m), 1266(s), 1174(m), 1105(m), 1071(s), 1007(s), 821(s), 747(s), 588(m), 559(m), 496(m), 454(m). ${ }^{1} \mathrm{H} \mathrm{NMR}\left(\mathrm{CD}_{2} \mathrm{Cl}_{2}\right.$, $\delta), 7.82-7.80(\mathrm{~m}, 2 \mathrm{H}, \mathrm{Ar}-\mathrm{H}), 7.78(\mathrm{~d}, J(\mathrm{H}, \mathrm{H})=6.9 \mathrm{~Hz}, 2 \mathrm{H}, \mathrm{Ar}-\mathrm{H})$, $7.61(\mathrm{~d}, J(\mathrm{H}, \mathrm{H})=6.9 \mathrm{~Hz}, 2 \mathrm{H}, \mathrm{Ar}-\mathrm{H}), 7.58-7.54(\mathrm{~m}, 3 \mathrm{H}, \mathrm{Ar}-\mathrm{H})$, 4.39-4.34 (m, 2H, CH), $2.54\left(\mathrm{~d}, 2 \mathrm{H}, \mathrm{SeCH}_{2}\right), 1.46(\mathrm{~d}, J(\mathrm{H}, \mathrm{H})=6.4$ $\left.\mathrm{H}, 12 \mathrm{H}, \mathrm{CH}_{3}\right) \mathrm{ppm} .{ }^{13} \mathrm{C}$ NMR $\left(\mathrm{CD}_{2} \mathrm{Cl}_{2}, \delta\right), 194.1(\mathrm{C}=\mathrm{O}), 133.5(\mathrm{~d}$, $J(\mathrm{P}, \mathrm{C})=112.1 \mathrm{~Hz}), 132.1,131.9,131.6(\mathrm{~d}, J(\mathrm{P}, \mathrm{C})=3.1 \mathrm{~Hz}), 131.2$ $(\mathrm{d}, J(\mathrm{P}, \mathrm{C})=12.5 \mathrm{~Hz}), 130.5,130.2,128.3(\mathrm{~d}, J(\mathrm{P}, \mathrm{C})=13.5 \mathrm{~Hz})$, 50.0, 39.3, 22.8 ppm. ${ }^{31} \mathrm{P}$ NMR $\left(\mathrm{CD}_{2} \mathrm{Cl}_{2}, \delta\right), 62.5(\mathrm{~s}, J(\mathrm{P}, \mathrm{Se})=366$ $\mathrm{Hz}, J(\mathrm{P}, \mathrm{Se})=789 \mathrm{~Hz}) \mathrm{ppm} .{ }^{77} \mathrm{Se} \mathrm{NMR}\left(\mathrm{CD}_{2} \mathrm{Cl}_{2}, \delta\right), 311.7(\mathrm{~d}$, $J(\mathrm{P}, \mathrm{Se})=367 \mathrm{~Hz}),-26.1(\mathrm{~d}, J(\mathrm{P}, \mathrm{Se})=789 \mathrm{~Hz}) \mathrm{ppm}$. Mass spectrum $\left[\mathrm{CI}^{+}, \mathrm{m} / \mathrm{z}\right]: 566[\mathrm{M}+\mathrm{H}]^{+}$. Accurate mass measurement $\left(\mathrm{CI}^{+}\right)$: 565.9269, calculate mass for $\mathrm{C}_{20} \mathrm{H}_{24} \mathrm{BrNOPSe}_{2} \mathrm{H}[\mathrm{M}+\mathrm{H}]^{+}$: 565.9266 .

\section{$N, N$-Diisobuyl}

$S e$-[2-(4-bromophenyl)-2-oxoethyl phenylphosphonamidodiselenoate (2l): $0.398 \mathrm{~g}$ as a reddish yellow sticky oil in $67 \%$ yield. Selected IR $\left(\mathrm{KBr}, \mathrm{cm}^{-1}\right)$ : $1683(\mathrm{~s})$, 1585(s), 1465(m), 1434(m), 1394(m), 1267(s), 1177(m), 1156(m), 1094(s), 1070(s), 1009(s), 876(m), 819(m), 745(s), 704(m), 690(m), $575(\mathrm{~s}), 490(\mathrm{~m}) .{ }^{1} \mathrm{H}$ NMR $\left(\mathrm{CD}_{2} \mathrm{Cl}_{2}, \delta\right), 8.02(\mathrm{dd}, J(\mathrm{P}, \mathrm{H})=14.6 \mathrm{~Hz}$ $J(\mathrm{H}, \mathrm{H})=6.6 \mathrm{~Hz}, 2 \mathrm{H}, \mathrm{Ar}-\mathrm{H}), 7.81(\mathrm{~d}, J(\mathrm{H}, \mathrm{H})=6.9 \mathrm{~Hz}, 2 \mathrm{H}, \mathrm{Ar}-\mathrm{H})$, $7.59(\mathrm{~d}, J(\mathrm{H}, \mathrm{H})=6.9 \mathrm{~Hz}, 2 \mathrm{H}, \mathrm{Ar}-\mathrm{H}), 7.51-7.42(\mathrm{~m}, 3 \mathrm{H}, \mathrm{Ar}-\mathrm{H})$, $4.27\left(\mathrm{~d}, J(\mathrm{P}, \mathrm{H})=10.8 \mathrm{~Hz}, 2 \mathrm{H}, \mathrm{SeCH}_{2}\right), 2.94-2.81\left(\mathrm{~m}, 4 \mathrm{H}, \mathrm{NCH}_{2}\right)$, 1.89-1.81 (m, 2H, CH), $0.81\left(\mathrm{~d}, J(\mathrm{H}, \mathrm{H})=6.6 \mathrm{H}, 6 \mathrm{H}, \mathrm{CH}_{3}\right), 0.75(\mathrm{~d}$, $\left.J(\mathrm{H}, \mathrm{H})=6.6 \mathrm{H}, 6 \mathrm{H}, \mathrm{CH}_{3}\right)$ ppm. ${ }^{13} \mathrm{C} \mathrm{NMR}\left(\mathrm{CD}_{2} \mathrm{Cl}_{2}, \delta\right), 193.9$ $(\mathrm{C}=\mathrm{O}), 135.2(\mathrm{~d}, J(\mathrm{P}, \mathrm{C})=112.1 \mathrm{~Hz}), 132.3,132.1,132.0(\mathrm{~d}, J(\mathrm{P}, \mathrm{C})$ $=3.1 \mathrm{~Hz}), 131.9,130.2(\mathrm{~d}, J(\mathrm{P}, \mathrm{C})=12.5 \mathrm{~Hz}), 129.5,128.3(\mathrm{~d}$ $J(\mathrm{P}, \mathrm{C})=13.5 \mathrm{~Hz}), 55.3,38.9,26.8,20.3, \mathrm{ppm} .{ }^{31} \mathrm{P} \mathrm{NMR}\left(\mathrm{CD}_{2} \mathrm{Cl}_{2}\right.$, $\delta), 78.4(\mathrm{~s}, J(\mathrm{P}, \mathrm{Se})=362 \mathrm{~Hz}, J(\mathrm{P}, \mathrm{Se})=798 \mathrm{~Hz}) \mathrm{ppm} .{ }^{77} \mathrm{Se} \mathrm{NMR}$ $\left(\mathrm{CD}_{2} \mathrm{Cl}_{2}, \delta\right), 299.8(\mathrm{~d}, J(\mathrm{P}, \mathrm{Se})=362 \mathrm{~Hz}),-93.5(\mathrm{~d}, J(\mathrm{P}, \mathrm{Se})=799$ $\mathrm{Hz}) \mathrm{ppm}$. Mass spectrum $\left[\mathrm{ES}^{+}, \mathrm{m} / \mathrm{z}\right]: 616[\mathrm{M}+\mathrm{Na}]^{+}$. Accurate mass measurement $\left(\mathrm{ES}^{+}\right)$: 615.9409, calculate mass for $\mathrm{C}_{22} \mathrm{H}_{29} \mathrm{NONaBrPSe}{ }_{2}[\mathrm{M}+\mathrm{Na}]^{+}: 615.9398$.

\section{$N, N$-Diisopropyl}

Se-[2-(hydroxymethyl)benzyl] phenylphosphonamidodiselenoate (2m): $0.215 \mathrm{~g}$ as a yellow paste in $44 \%$ yield. Selected IR $\left(\mathrm{KBr}, \mathrm{cm}^{-1}\right): 1486(\mathrm{~m}), 1453(\mathrm{~m})$, 1435(m), 1172(m), 1040(m), 759(s), 711(m), 550(s), 507(m), 442(m). ${ }^{1} \mathrm{H}$ NMR $\left(\mathrm{CD}_{2} \mathrm{Cl}_{2}, \delta\right), 8.01-7.94(\mathrm{~m}, 2 \mathrm{H}, \mathrm{Ar}-\mathrm{H}), 7.35-7.22$ $(\mathrm{m}, 7 \mathrm{H}, \mathrm{Ar}-\mathrm{H}), 4.67\left(\mathrm{~d}, J(\mathrm{H}, \mathrm{H})=15.4 \mathrm{~Hz}, 2 \mathrm{H}, \mathrm{CH}_{2}\right), 3.96-3.85(\mathrm{~m}$, $2 \mathrm{H}, \mathrm{CH}), 3.70(\mathrm{t}, 1 \mathrm{H}, \mathrm{OH}), 2.59$ (d, $\left.2 \mathrm{H}, \mathrm{SeCH}_{2}\right), 1.29(\mathrm{dd}, 12 \mathrm{H}$, $\left.\mathrm{CH}_{3}\right)$ ppm. ${ }^{13} \mathrm{C}$ NMR $\left(\mathrm{CD}_{2} \mathrm{Cl}_{2}, \delta\right), 139.2,136.7,133.5,131.5$, 131.2, 131.0, 130.3, 128.8, 127.7, 127.3, 62.6, 50.0, 29.9, 22.8 ppm. ${ }^{31} \mathrm{P} \mathrm{NMR}\left(\mathrm{CD}_{2} \mathrm{Cl}_{2}, \delta\right), 59.9(\mathrm{~s}, J(\mathrm{P}, \mathrm{Se})=387 \mathrm{~Hz}, J(\mathrm{P}, \mathrm{Se})=777 \mathrm{~Hz})$ ppm. ${ }^{77} \mathrm{Se} \mathrm{NMR}\left(\mathrm{CD}_{2} \mathrm{Cl}_{2}, \delta\right), 361.4(\mathrm{~d}, J(\mathrm{P}, \mathrm{Se})=388 \mathrm{~Hz}),-26.4(\mathrm{~d}$, $J(\mathrm{P}, \mathrm{Se})=777 \mathrm{~Hz}) \mathrm{ppm}$. Mass spectrum $\left[\mathrm{CI}^{+}, \mathrm{m} / \mathrm{z}\right]: 490[\mathrm{M}+\mathrm{H}]^{+}$ Accurate mass measurement $\left(\mathrm{CI}^{+}\right): 490.0308$, calculate mass for $\mathrm{C}_{20} \mathrm{H}_{28} \mathrm{NOPSe}_{2} \mathrm{H}[\mathrm{M}+\mathrm{H}]^{+}: 490.0315$.

$N$-Benzyl

Se-[2-(4-bromophenyl)-2-oxoethyl] phenylphosphonamidodiselenoate (2n): $0.534 \mathrm{~g}$ as a yellow paste in $47 \%$ yield. Selected IR $\left(\mathrm{KBr}, \mathrm{cm}^{-1}\right): 1693(\mathrm{vs}), 1580(\mathrm{~s}), 1479(\mathrm{~m})$, 1453(m), 1434(m), 1394(m), 1274(m), 1189(m), 1100(m), 1056(s), 984(s), 801(s), 731(s), 686(s), 555(s), 492(m), 475(m), 445(m). ${ }^{1} \mathrm{H}$ $\operatorname{NMR}\left(\mathrm{CD}_{2} \mathrm{Cl}_{2}, \delta\right), 8.03(\mathrm{~d}, J(\mathrm{H}, \mathrm{H})=8.26 \mathrm{~Hz}, 2 \mathrm{H}, \mathrm{Ar}-\mathrm{H}), 7.81-7.77$ (m, 2H, Ar-H), 7.60-7.47 (m, 5H, Ar-H), 7.36-7.26 (m, 5H, Ar-H), 4.31-4.01 (m, 4H, $\left.\mathrm{CH}_{2}\right), 3.68-3.60(\mathrm{~m}, 1 \mathrm{H}, \mathrm{NH})$ ppm. ${ }^{13} \mathrm{C}$ NMR $\left(\mathrm{CD}_{2} \mathrm{Cl}_{2}, \delta\right), 193.9(\mathrm{C}=\mathrm{O}), 138.7,138.5,134.8(\mathrm{~d}, J(\mathrm{P}, \mathrm{C})=91.3$ $\mathrm{Hz}), 132.6(\mathrm{~d}, J(\mathrm{P}, \mathrm{C})=3.1 \mathrm{~Hz}), 132.1,130.9(\mathrm{~d}, J(\mathrm{P}, \mathrm{C})=12.5 \mathrm{~Hz})$, $130.4(\mathrm{~d}, J(\mathrm{P}, \mathrm{C})=13.5 \mathrm{~Hz}), 129.9,128.9,128.7,128.1,127.6,46.9$, 37.2 ppm. ${ }^{31} \mathrm{P}$ NMR $\left(\mathrm{CD}_{2} \mathrm{Cl}_{2}, \delta\right), 58.3(\mathrm{~s}, J(\mathrm{P}, \mathrm{Se})=376 \mathrm{~Hz}, J(\mathrm{P}, \mathrm{Se})$ $=798 \mathrm{~Hz}) \mathrm{ppm} .{ }^{77} \mathrm{Se} \mathrm{NMR}\left(\mathrm{CD}_{2} \mathrm{Cl}_{2}, \delta\right), 324.8(\mathrm{~d}, J(\mathrm{P}, \mathrm{Se})=376$ $\mathrm{Hz}),-103.4(\mathrm{~d}, J(\mathrm{P}, \mathrm{Se})=798 \mathrm{~Hz}) \mathrm{ppm}$. Mass spectrum $\left[\mathrm{ES}^{+}, \mathrm{m} / \mathrm{z}\right]$ : $594[\mathrm{M}+\mathrm{Na}]^{+}$. Accurate mass measurement $\left(\mathrm{CI}^{+}\right)$: 571.8787, calculate mass for $\mathrm{C}_{21} \mathrm{H}_{19} \mathrm{NOPSe} \mathrm{BrH}_{2}[\mathrm{M}+\mathrm{H}]^{+}: 571.8790$.

\section{General procedure for synthesis of compounds 3a-n: A mixture} of amine $(4.0 \mathrm{mmol})$ and WR $(0.54 \mathrm{~g}, 1.0 \mathrm{mmol})$ in tetrahydrofuran $(60 \mathrm{~mL})$ was stirred at room temperature for $2 \mathrm{~h}$. The brown suspension disappeared and a pale yellow suspension formed. To the mixture, the appropriate dihaloalkane $(1.0 \mathrm{mmol})$ was added and the mixture was allowed to stir at room temperature for $24 \mathrm{~h}$. After filtering to remove insoluble solid and drying in $v a c u o$, the residue was dissolved in dichloromethane $(c a .2 .0 \mathrm{~mL})$ and purified by column chromatography on silica gel using dichloromethane as eluent to give the compounds 3a-n.

\section{Methylene}

bis $(N$-cyclohexyl- $P$ -

phenylphosphonamidodiselenoate) (3a): $0.585 \mathrm{~g}$ as a pale yellow oil in $79 \%$ yield. A pair of diastereoisomers was found in multiNMR spectra in $c a .1: 1$ intensity ratio. Selected IR $\left(\mathrm{KBr}, \mathrm{cm}^{-1}\right)$ : 1435(s), 1403(m), 1292(m), 1231(m), 1139(m), 1077(vs), 994(s), 902(s), 879(s), 745(s), 688(s), 567(s), 518(vs), 490(s), 438(m). ${ }^{1} \mathrm{H}$ NMR $\left(\mathrm{CD}_{2} \mathrm{Cl}_{2}, \delta\right), 8.01-7.88$ (m, 2x4H, Ar-H), 7.60-7.39 (m, 2x6H, 
Ar-H), 4.42-4.20 (m, 2x2H, Cyclohexyl-H), 3.21 (d, 2H, $\mathrm{SeCH}_{2}$ ), $3.05\left(\mathrm{~d}, 2 \mathrm{H}, \mathrm{SeCH}_{2}\right), 1.96-0.83(\mathrm{~m}, 2 \mathrm{x} 20 \mathrm{H}, \mathrm{NH}+$ Cyclohexyl-H) ppm. ${ }^{13} \mathrm{C}$ NMR $\left(\mathrm{CD}_{2} \mathrm{Cl}_{2}, \delta\right), 136.2(\mathrm{~d}, J(\mathrm{P}, \mathrm{C})=88.2 \mathrm{~Hz}), 132.3(\mathrm{~d}$, $J(\mathrm{P}, \mathrm{C})=3.1 \mathrm{~Hz}), 130.9(\mathrm{~d}, J(\mathrm{P}, \mathrm{C})=12.5 \mathrm{~Hz}), 128.6(\mathrm{~d}, J(\mathrm{P}, \mathrm{C})=$ $13.5 \mathrm{~Hz}), 53.1(\mathrm{~d}, J(\mathrm{P}, \mathrm{C})=9.5 \mathrm{~Hz}), 35.6,29.1,25.4,25.2 \mathrm{ppm} .{ }^{31} \mathrm{P}$ $\operatorname{NMR}\left(\mathrm{CD}_{2} \mathrm{Cl}_{2}, \delta\right), 54.9(\mathrm{~s}, J(\mathrm{P}, \mathrm{Se})=374 \mathrm{~Hz}, J(\mathrm{P}, \mathrm{Se})=791 \mathrm{~Hz})$, $54.8(\mathrm{~s}, J(\mathrm{P}, \mathrm{Se})=371 \mathrm{~Hz}, J(\mathrm{P}, \mathrm{Se})=791 \mathrm{~Hz}) \mathrm{ppm} .{ }^{77} \mathrm{Se} \mathrm{NMR}$ $\left(\mathrm{CD}_{2} \mathrm{Cl}_{2}, \delta\right), 398.6(\mathrm{~d}, J(\mathrm{P}, \mathrm{Se})=371 \mathrm{~Hz}), 395.5(\mathrm{~d}, J(\mathrm{P}, \mathrm{Se})=374$ $\mathrm{Hz}),-91.9(\mathrm{~d}, J(\mathrm{P}, \mathrm{Se})=791 \mathrm{~Hz}),-92.7(\mathrm{~d}, J(\mathrm{P}, \mathrm{Se})=791 \mathrm{~Hz}) \mathrm{ppm}$. Mass spectrum $\left[\mathrm{CI}^{+}, \mathrm{m} / \mathrm{z}\right]: 745[\mathrm{M}+\mathrm{H}]^{+}$. Accurate mass measurement $\left(\mathrm{CI}^{+} \mathrm{MS}\right)$ : 744.9099, calculate mass for $\mathrm{C}_{25} \mathrm{H}_{37} \mathrm{~N}_{2} \mathrm{P}_{2} \mathrm{Se}_{4}[\mathrm{M}+\mathrm{H}]: 744.9101$.

\section{Ethane-1,2-diyl}

$\operatorname{bis}(N$-cyclohexyl- $P$ phenylphosphonamidodiselenoate) (3b): $0.604 \mathrm{~g}$ as a pale yellow oil in $80 \%$ yield. A pair of diastereoisomers was found in multiNMR spectra in ca. $1: 1$ intensity ratio. Selected $\mathrm{IR}\left(\mathrm{KBr}, \mathrm{cm}^{-1}\right)$ : 1434(m), 1405(m), 1077(s), 994(m), 878(m), 745(m), 688(s), 519(s), 479(m), 443(m). ${ }^{1} \mathrm{H}$ NMR $\left(\mathrm{CD}_{2} \mathrm{Cl}_{2}, \delta\right), 8.12-7.90(\mathrm{~m}, 2 \mathrm{x} 4 \mathrm{H}$, $\mathrm{Ar}-\mathrm{H}), \quad 7.50-7.43 \quad(\mathrm{~m}, \quad 2 \times 6 \mathrm{H}, \quad \mathrm{Ar}-\mathrm{H}), \quad 4.36-4.06 \quad(\mathrm{dd}, 2 \times 2 \mathrm{H}$, Cyclohexyl-H), 3.20-3.08 (m, 2x4H, $\left.\mathrm{CH}_{2} \mathrm{Se}\right), 2.28-1.17$ (m, $2 \times 22 \mathrm{H}$ $\mathrm{NH}+$ Cyclohexyl-H) ppm. ${ }^{13} \mathrm{C}$ NMR $\left(\mathrm{CD}_{2} \mathrm{Cl}_{2}, \delta\right), 135.7(\mathrm{~d}, J(\mathrm{P}, \mathrm{C})$ $=90.3 \mathrm{~Hz}), 135.6(\mathrm{~d}, J(\mathrm{P}, \mathrm{C})=90.3 \mathrm{~Hz}), 132.2(\mathrm{~d}, J(\mathrm{P}, \mathrm{C})=3.1 \mathrm{~Hz})$, $132.5(\mathrm{~d}, J(\mathrm{P}, \mathrm{C})=3.1 \mathrm{~Hz}), 130.9(\mathrm{~d}, J(\mathrm{P}, \mathrm{C})=12.5 \mathrm{~Hz}), 130.8(\mathrm{~d}$, $J(\mathrm{P}, \mathrm{C})=12.5 \mathrm{~Hz}), 128.6130 .9(\mathrm{~d}, J(\mathrm{P}, \mathrm{C})=13.5 \mathrm{~Hz}), 53.0,52.9$, 35.7, 35.6, 32.1, 30.9, 25.4, 25.3 ppm. ${ }^{31} \mathrm{P}$ NMR $\left(\mathrm{CD}_{2} \mathrm{Cl}_{2}, \delta\right), 52.5$ $(\mathrm{s}, J(\mathrm{P}, \mathrm{Se})=384 \mathrm{~Hz}, J(\mathrm{P}, \mathrm{Se})=784 \mathrm{~Hz}), 52.4(\mathrm{~s}, J(\mathrm{P}, \mathrm{Se})=386 \mathrm{~Hz}$, $J(\mathrm{P}, \mathrm{Se})=786 \mathrm{~Hz}) \mathrm{ppm} .{ }^{77} \mathrm{Se} \mathrm{NMR}\left(\mathrm{CD}_{2} \mathrm{Cl}_{2}, \delta\right), 378.5(\mathrm{~d}, J(\mathrm{P}, \mathrm{Se})=$ $384 \mathrm{~Hz}), 375.6(\mathrm{~d}, J(\mathrm{P}, \mathrm{Se})=386 \mathrm{~Hz}),-90.1(\mathrm{~d}, J(\mathrm{P}, \mathrm{Se})=784 \mathrm{~Hz})$, $94.7(\mathrm{~d}, J(\mathrm{P}, \mathrm{Se})=786 \mathrm{~Hz}) \mathrm{ppm}$. Mass spectrum $\left[\mathrm{CI}^{+}, \mathrm{m} / \mathrm{z}\right]: 759$ $[\mathrm{M}+\mathrm{H}]^{+}$. Accurate mass measurement $\left(\mathrm{CI}^{+} \mathrm{MS}\right)$ : 758.9260, calculate mass for $\mathrm{C}_{26} \mathrm{H}_{39} \mathrm{~N}_{2} \mathrm{P}_{2} \mathrm{Se}_{4}[\mathrm{M}+\mathrm{H}]$ : 758.9256 .

\section{Propane-1,3-diyl}

bis $(N$-cyclohexyl-P phenylphosphonamidodiselenoate) (3c): $0.540 \mathrm{~g}$ as a pale yellow paste in $70 \%$ yield. A pair of diastereoisomers was found in multiNMR spectra in ca. 1: 1 intensity ratio. Selected IR $\left(\mathrm{KBr}, \mathrm{cm}^{-1}\right)$ : 1435(m), 1403(m), 1289(m), 1229(m), 1078(vs), 994(m), 878(m), 745(s), 689(s), 568)(m), 520(s), 489(m). ${ }^{1} \mathrm{H}$ NMR $\left(\mathrm{CD}_{2} \mathrm{Cl}_{2}, \delta\right)$, 8.03-7.95 (m, 2x4H, Ar-H), 7.53-7.451 (m, 2x6H, Ar-H), 3.28-3.11 $\left(\mathrm{m}, 2 \times 2 \mathrm{H}\right.$, Cyclohexyl-H), 2.96-2.76 (m, 2x6H, $\left.\mathrm{SeCH}_{2}+\mathrm{NH}\right)$, 2.14-1.07 (m, 2x22H, Cyclohexyl-H) ppm. ${ }^{13} \mathrm{C} \mathrm{NMR}\left(\mathrm{CD}_{2} \mathrm{Cl}_{2}, \delta\right)$, $137.1(\mathrm{~d}, J(\mathrm{P}, \mathrm{C})=88.2 \mathrm{~Hz}), 132.0(\mathrm{~d}, J(\mathrm{P}, \mathrm{C})=3.1 \mathrm{~Hz}), 130.8(\mathrm{~d}$, $J(\mathrm{P}, \mathrm{C})=12.5 \mathrm{~Hz}), 128.5(\mathrm{~d}, J(\mathrm{P}, \mathrm{C})=13.5 \mathrm{~Hz}), 53.0,35.6,32.3$, 31.2, 25.4, 25.3 ppm. ${ }^{31} \mathrm{P}$ NMR $\left(\mathrm{CD}_{2} \mathrm{Cl}_{2}, \delta\right), 53.7(\mathrm{~s}, J(\mathrm{P}, \mathrm{Se})=384$ $\mathrm{Hz}, J(\mathrm{P}, \mathrm{Se})=789 \mathrm{~Hz}), 53.6(\mathrm{~s}, J(\mathrm{P}, \mathrm{Se})=386 \mathrm{~Hz}, J(\mathrm{P}, \mathrm{Se})=786$ $\mathrm{Hz})$ ppm. ${ }^{77} \mathrm{Se} \mathrm{NMR}\left(\mathrm{CD}_{2} \mathrm{Cl}_{2}, \delta\right), 325.1(\mathrm{~d}, J(\mathrm{P}, \mathrm{Se})=384 \mathrm{~Hz})$, $324.8(\mathrm{~d}, J(\mathrm{P}, \mathrm{Se})=386 \mathrm{~Hz}),-102.8(\mathrm{~d}, J(\mathrm{P}, \mathrm{Se})=789 \mathrm{~Hz}),-103.5$ $(\mathrm{d}, J(\mathrm{P}, \mathrm{Se})=786 \mathrm{~Hz}) \mathrm{ppm}$. Mass spectrum $\left[\mathrm{CI}^{+}, \mathrm{m} / \mathrm{z}\right]: 773[\mathrm{M}+\mathrm{H}]^{+}$ Accurate mass measurement $\left(\mathrm{CI}^{+} \mathrm{MS}\right)$ : 772.9416 , calculate mass for $\mathrm{C}_{27} \mathrm{H}_{41} \mathrm{~N}_{2} \mathrm{P}_{2} \mathrm{Se}_{4}[\mathrm{M}+\mathrm{H}]$ : 772.9421 .

\section{Butane-1,4-diyl}

bis $(N$-cyclohexyl-P. phenylphosphonamidodiselenoate) (3d): $0.610 \mathrm{~g}$ as a pale green paste in $48 \%$ yield. A pair of diastereoisomers was found in multiNMR spectra in ca. 1: 1 intensity ratio. Selected IR $\left(\mathrm{KBr}, \mathrm{cm}^{-1}\right)$ : 1436(m), 1396(m), 1078(s), 997(m), 886(m), 746(s), 688(s), 572(m), 519(s), 485(m). ${ }^{1} \mathrm{H}$ NMR $\left(\mathrm{CD}_{2} \mathrm{Cl}_{2}, \delta\right), 7.98-7.96(\mathrm{~m}, 2 \mathrm{x} 4 \mathrm{H}$, Ar-H), 7.47-7.42 (m, 2x6H, Ar-H), 3.96-3.79 (m, 2x4H, $\mathrm{SeCH}_{2}$ ), 2.81-1.15 (m, 2x28H, NH + Cyclohexyl-H) ppm. ${ }^{13} \mathrm{C} \mathrm{NMR}$ $\left(\mathrm{CD}_{2} \mathrm{Cl}_{2}, \delta\right), 137.1(\mathrm{~d}, J(\mathrm{P}, \mathrm{C})=86.2 \mathrm{~Hz}), 132.7(\mathrm{~d}, J(\mathrm{P}, \mathrm{C})=3.1$ $\mathrm{Hz}), 130.8(\mathrm{~d}, J(\mathrm{P}, \mathrm{C})=12.5 \mathrm{~Hz}), 128.4(\mathrm{~d}, J(\mathrm{P}, \mathrm{C})=13.5 \mathrm{~Hz}), 52.9$, 35.7, 33.4, 25.9, 25.1, 24.5 ppm. ${ }^{31} \mathrm{P}$ NMR $\left(\mathrm{CD}_{2} \mathrm{Cl}_{2}, \delta\right), 53.5(\mathrm{~s}$, $J(\mathrm{P}, \mathrm{Se})=384 \mathrm{~Hz}, J(\mathrm{P}, \mathrm{Se})=789 \mathrm{~Hz}), 53.4(\mathrm{~s}, J(\mathrm{P}, \mathrm{Se})=386 \mathrm{~Hz}$, $J(\mathrm{P}, \mathrm{Se})=786 \mathrm{~Hz}) \mathrm{ppm} .{ }^{77} \mathrm{Se} \mathrm{NMR}\left(\mathrm{CD}_{2} \mathrm{Cl}_{2}, \delta\right), 326.7(\mathrm{~d}, J(\mathrm{P}, \mathrm{Se})=$ $386 \mathrm{~Hz}), 326.3(\mathrm{~d}, J(\mathrm{P}, \mathrm{Se})=388 \mathrm{~Hz}),-105.0(\mathrm{~d}, J(\mathrm{P}, \mathrm{Se})=789 \mathrm{~Hz})$, $-105.2(\mathrm{~d}, J(\mathrm{P}, \mathrm{Se})=786 \mathrm{~Hz}) \mathrm{ppm}$. Mass spectrum $\left[\mathrm{CI}^{+}, \mathrm{m} / \mathrm{z}\right]: 789$ $[\mathrm{M}+\mathrm{H}]^{+}$. Accurate mass measurement $\left(\mathrm{C} \mid \mathrm{I}^{+} \mathrm{MS}\right)$ : 788.9556, calculate mass for $\mathrm{C}_{28} \mathrm{H}_{43} \mathrm{~N}_{2} \mathrm{PSe}_{4}[\mathrm{M}+\mathrm{H}]: 788.9562$.
1,2-Phenylenebis(methylene) $\quad$ bis $(N-\quad$ cyclohexyl-Pphenylphosphonamidodiselenoate) (3e): $0.642 \mathrm{~g}$ as a milky green solid in $77 \%$ yield. A pair of diastereoisomers was found in multiNMR spectra in ca. 1: 1 intensity ratio. Selected IR $\left(\mathrm{KBr}, \mathrm{cm}^{-1}\right)$ : 1435(m), 1401(m), 1290(w), 1072(m), 1076(s), 993(m), 877(m), 745(s), 688(s), 567(m), 519(s), 489(m), m436(m). ${ }^{1} \mathrm{H} \quad \mathrm{NMR}$ $\left(\mathrm{CD}_{2} \mathrm{Cl}_{2}, \delta\right), 7.94-7.88(\mathrm{~m}, 2 \times 4 \mathrm{H}, \mathrm{Ar}-\mathrm{H}), 7.38-7.22(\mathrm{~m}, 2 \times 6 \mathrm{H}, \mathrm{Ar}-$ H), 7.21-6.99 (m, 2x4H, Ar-H), 4.38-4.18 (m, 2x2H, Cyclohexyl$\mathrm{H})$, 3.11-2.76 (m, 2x6H, $\left.\mathrm{SeCH}_{2}+\mathrm{NH}\right), 1.86-1.12(\mathrm{~m}, 2 \times 20 \mathrm{H}$, Cyclohexyl-H) ppm. ${ }^{13} \mathrm{C}$ NMR $\left(\mathrm{CD}_{2} \mathrm{Cl}_{2}, \delta\right), 137.3,136.6,136.1$, 132.0, 130.9, 130.8, 128.6, 128.4, 127.8, 53.1, 52.7, 35.6, 33.8, 33.4, 25.4, 25.2 ppm. ${ }^{31} \mathrm{P}$ NMR $\left(\mathrm{CD}_{2} \mathrm{Cl}_{2}, \delta\right), 53.9(\mathrm{~s}, J(\mathrm{P}, \mathrm{Se})=386$ $\mathrm{Hz}, J(\mathrm{P}, \mathrm{Se})=789 \mathrm{~Hz}), 53.8(\mathrm{~s}, J(\mathrm{P}, \mathrm{Se})=386 \mathrm{~Hz}, J(\mathrm{P}, \mathrm{Se})=789$ $\mathrm{Hz})$ ppm. ${ }^{77} \mathrm{Se} \mathrm{NMR}\left(\mathrm{CD}_{2} \mathrm{Cl}_{2}, \delta\right), 395.9(\mathrm{~d}, J(\mathrm{P}, \mathrm{Se})=386 \mathrm{~Hz})$, $395.1(\mathrm{~d}, J(\mathrm{P}, \mathrm{Se})=386 \mathrm{~Hz}),-96.7(\mathrm{~d}, J(\mathrm{P}, \mathrm{Se})=789 \mathrm{~Hz}),-96.8(\mathrm{~d}$, $J(\mathrm{P}, \mathrm{Se})=789 \mathrm{~Hz})$ ppm. Mass spectrum $\left[\mathrm{CI}^{+}, \mathrm{m} / \mathrm{z}\right]: 835[\mathrm{M}+\mathrm{H}]^{+}$. Accurate mass measurement $\left(\mathrm{C} \mid \mathrm{I}^{+} \mathrm{MS}\right): 834.9569$, calculate mass for $\mathrm{C}_{32} \mathrm{H}_{43} \mathrm{~N}_{2} \mathrm{PSe}_{4}[\mathrm{M}+\mathrm{H}]: 834.9580$.

\section{Ethane-1,2-diyl}

bis $(N$-isopropyl- $P$ phenylphosphonamidodiselenoate) (3f): $0.440 \mathrm{~g}$ as a reddish pink paste in 55\% yield. A pair of diastereoisomers was found in multi-NMR spectra in ca. 2: 3 intensity ratio. Selected IR $(\mathrm{KBr}$, $\left.\mathrm{cm}^{-1}\right)$ : 1436(s), 1398(m), 1160(m), 1130(s), 1027(s), 946(m), 890(m), 747(s), 692(s), 586(s), 537(s). ${ }^{1} \mathrm{H}$ NMR $\left(\mathrm{CD}_{2} \mathrm{Cl}_{2}, \delta\right), 7.88-$ $7.79(\mathrm{~m}, 2 \times 4 \mathrm{H}, \mathrm{Ar}-\mathrm{H}), 7.50-7.43(\mathrm{~m}, 2 \times 6 \mathrm{H}, \mathrm{Ar}-\mathrm{H}), 4.25-4.05(\mathrm{~m}$, $2 \times 2 \mathrm{H}, \mathrm{CH}), 3.60-3.57\left(\mathrm{~m}, 2 \times 4 \mathrm{H}, \mathrm{SeCH}_{2}\right), 3.19-3.16(\mathrm{~m}, 2 \times 2 \mathrm{H}$, $\mathrm{NH}), 1.38-1.34\left(\mathrm{~m}, 2 \mathrm{x} 12 \mathrm{H}, \mathrm{CH}_{3}\right)$ ppm. ${ }^{13} \mathrm{C} \mathrm{NMR}\left(\mathrm{CD}_{2} \mathrm{Cl}_{2}, \delta\right)$, $137.1,136.5,131.6(\mathrm{~d}, J(\mathrm{P}, \mathrm{C})=3.1 \mathrm{~Hz}), 130.8,130.6,128.3,128.1$, 61.9, 61.8, 45.3, 44.7, 25.2, 24.7 ppm. ${ }^{31} \mathrm{P}$ NMR $\left(\mathrm{CD}_{2} \mathrm{Cl}_{2}, \delta\right), 53.0$ $(\mathrm{s}, J(\mathrm{P}, \mathrm{Se})=383 \mathrm{~Hz}, J(\mathrm{P}, \mathrm{Se})=786 \mathrm{~Hz}), 52.7(\mathrm{~s}, J(\mathrm{P}, \mathrm{Se})=385 \mathrm{~Hz}$, $J(\mathrm{P}, \mathrm{Se})=784 \mathrm{~Hz}) \mathrm{ppm} .{ }^{77} \mathrm{Se} \mathrm{NMR}\left(\mathrm{CD}_{2} \mathrm{Cl}_{2}, \delta\right), 514.7(\mathrm{~d}, J(\mathrm{P}, \mathrm{Se})=$ $385 \mathrm{~Hz}), 514.5(\mathrm{~d}, J(\mathrm{P}, \mathrm{Se})=383 \mathrm{~Hz}),-248.5(\mathrm{~d}, J(\mathrm{P}, \mathrm{Se})=784 \mathrm{~Hz})$, $-248.7(\mathrm{~d}, J(\mathrm{P}, \mathrm{Se})=786 \mathrm{~Hz}) \mathrm{ppm}$. Mass spectrum $\left[\mathrm{CI}^{+}, \mathrm{m} / \mathrm{z}\right]: 679$ $[\mathrm{M}+\mathrm{H}]^{+}$. Accurate mass measurement $\left(\mathrm{C} \mid \mathrm{I}^{+} \mathrm{MS}\right)$ : 678.8625, calculate mass for $\mathrm{C}_{20} \mathrm{H}_{31} \mathrm{~N}_{2} \mathrm{PSe}_{4}[\mathrm{M}+\mathrm{H}]$ : 678.8631 .

\section{Propane-1,3-diyl}

bis( $N$-isopropyl- $P$ phenylphosphonamidodiselenoate) (3g): $0.380 \mathrm{~g}$ as a reddish yellow paste in 55\% yield. A pair of diastereoisomers was found in multi-NMR spectra in ca. 1: 1 intensity ratio. Selected IR $(\mathrm{KBr}$, $\left.\mathrm{cm}^{-1}\right)$ : 163(w), 1435(m), 1285(m), 1114(m), 1029(s), 893(s), 806(s), 747(m), 691(s), 542(m). ${ }^{1} \mathrm{H}$ NMR $\left(\mathrm{CD}_{2} \mathrm{Cl}_{2}, \delta\right), 8.03-7.94(\mathrm{~m}, 2 \mathrm{x} 4 \mathrm{H}$, Ar-H), 7.54-7.47 (m, 2x6H, Ar-H), 3.90-3.52 (m, 2x2H, CH), 3.30$3.24\left(\mathrm{~m}, 2 \times 4 \mathrm{H}, \mathrm{SeCH}_{2}\right), 2.98-2.88(\mathrm{~m}, 2 \times 2 \mathrm{H}, \mathrm{NH}), 1.52-1.37(\mathrm{~m}$, $\left.2 \times 2 \mathrm{H}, \mathrm{CH}_{2}\right), 1.17-1.12\left(\mathrm{~m}, 2 \times 12 \mathrm{H}, \mathrm{CH}_{3}\right) \mathrm{ppm} .{ }^{13} \mathrm{C} \mathrm{NMR}\left(\mathrm{CD}_{2} \mathrm{Cl}_{2}\right.$, $\delta), 136.8,136.2,135.5,135.1,132.6(\mathrm{~d}, J(\mathrm{P}, \mathrm{C})=3.1 \mathrm{~Hz}), 132.1(\mathrm{~d}$, $J(\mathrm{P}, \mathrm{C})=3.1 \mathrm{~Hz}), 131.1,130.9,130.7,130.5,129.1,128.9,18.6$, $128.4,46.2,45.8,29.1,29.0,25.0,24.5,20.9$ ppm. ${ }^{31} \mathrm{P}$ NMR $\left(\mathrm{CD}_{2} \mathrm{Cl}_{2}, \delta\right), 53.9(\mathrm{~s}, J(\mathrm{P}, \mathrm{Se})=385 \mathrm{~Hz}, J(\mathrm{P}, \mathrm{Se})=789 \mathrm{~Hz}), 53.8(\mathrm{~s}$, $J(\mathrm{P}, \mathrm{Se})=385 \mathrm{~Hz}, J(\mathrm{P}, \mathrm{Se})=789 \mathrm{~Hz}) \mathrm{ppm} .{ }^{77} \mathrm{Se} \mathrm{NMR}\left(\mathrm{CD}_{2} \mathrm{Cl}_{2}, \delta\right)$, $323.4(\mathrm{~d}, J(\mathrm{P}, \mathrm{Se})=385 \mathrm{~Hz}), 323.0(\mathrm{~d}, J(\mathrm{P}, \mathrm{Se})=385 \mathrm{~Hz}),-164.2(\mathrm{~d}$, $J(\mathrm{P}, \mathrm{Se})=789 \mathrm{~Hz}),-173.8(\mathrm{~d}, J(\mathrm{P}, \mathrm{Se})=786 \mathrm{~Hz})$ ppm. Mass spectrum $\left[\mathrm{CI}^{+}, \mathrm{m} / \mathrm{z}\right]: 693[\mathrm{M}+\mathrm{H}]^{+}$. Accurate mass measurement (C|I ${ }^{+} \mathrm{MS}$ ): 692.8779, calculate mass for $\mathrm{C}_{21} \mathrm{H}_{33} \mathrm{~N}_{2} \mathrm{PSe}_{4}[\mathrm{M}+\mathrm{H}]$ : 692.8788 .

\section{1,2-Phenylenebis(methylene)}

bis $(N$-isopropyl- $P$ phenylphosphonamidodiselenoate) (3h): $0.495 \mathrm{~g}$ as a pale green paste in $66 \%$ yield. A pair of diastereoisomers was found in multiNMR spectra in ca. $3: 2$ intensity ratio. Selected IR $\left(\mathrm{KBr}, \mathrm{cm}^{-1}\right)$ : 1434(m), 1362(m), 1124(m), 1096(m), 1007(s), 881(s), 744(s), 688(s), 532(s). ${ }^{1} \mathrm{H}$ NMR $\left(\mathrm{CD}_{2} \mathrm{Cl}_{2}, \delta\right), 8.04-7.94(\mathrm{~m}, 2 \mathrm{x} 4 \mathrm{H}, \mathrm{Ar}-\mathrm{H})$, 7.52-7.47 (m, 2x6H, Ar-H), 7.15-7.06 (m, $2 \times 4 \mathrm{H}, \mathrm{Ar}-\mathrm{H}), 4.27-4.20$ (m, $2 \times 2 \mathrm{H}, \mathrm{CH}), 3.92-3.83\left(\mathrm{~m}, 2 \times 4 \mathrm{H}, \mathrm{SeCH}_{2}\right), 2.74-2.68(\mathrm{~m}, 2 \times 2 \mathrm{H}$, $\mathrm{NH})$, $1.15-1.08\left(\mathrm{~m}, 2 \times 12 \mathrm{H}, \mathrm{CH}_{3}\right)$ ppm. ${ }^{13} \mathrm{C} \mathrm{NMR}\left(\mathrm{CD}_{2} \mathrm{Cl}_{2}, \delta\right)$, 137.6, 137.1, 136.6, 135.8, $132.2(\mathrm{~d}, J(\mathrm{P}, \mathrm{C})=3.1 \mathrm{~Hz}), 131.0(\mathrm{~d}$, $J(\mathrm{P}, \mathrm{C})=12.5 \mathrm{~Hz}), 130.9(\mathrm{~d}, J(\mathrm{P}, \mathrm{C})=12.5 \mathrm{~Hz}), 128.6(\mathrm{~d}, J(\mathrm{P}, \mathrm{C})=$ $13.5 \mathrm{~Hz}), 127.8(\mathrm{~d}, J(\mathrm{P}, \mathrm{C})=13.5 \mathrm{~Hz}), 46.3,33.7,24.9,24.8 \mathrm{ppm}$. ${ }^{31} \mathrm{P}$ NMR $\left(\mathrm{CD}_{2} \mathrm{Cl}_{2}, \delta\right), 54.2(\mathrm{~s}, J(\mathrm{P}, \mathrm{Se})=385 \mathrm{~Hz}, \quad J(\mathrm{P}, \mathrm{Se})=786$ 
$\mathrm{Hz}), 54.1(\mathrm{~s}, J(\mathrm{P}, \mathrm{Se})=385 \mathrm{~Hz}, J(\mathrm{P}, \mathrm{Se})=786 \mathrm{~Hz}) \mathrm{ppm} .{ }^{77} \mathrm{Se} \mathrm{NMR}$ $\left(\mathrm{CD}_{2} \mathrm{Cl}_{2}, \delta\right), 392.7(\mathrm{~d}, J(\mathrm{P}, \mathrm{Se})=385 \mathrm{~Hz}), 392.1(\mathrm{~d}, J(\mathrm{P}, \mathrm{Se})=385$ $\mathrm{Hz}),-99.5(\mathrm{~d}, J(\mathrm{P}, \mathrm{Se})=786 \mathrm{~Hz}),-99.7(\mathrm{~d}, J(\mathrm{P}, \mathrm{Se})=786 \mathrm{~Hz}) \mathrm{ppm}$. Mass spectrum $\left[\mathrm{CI}^{+}, \mathrm{m} / \mathrm{z}\right]: 755[\mathrm{M}+\mathrm{H}]^{+}$. Accurate mass

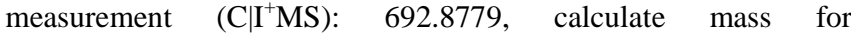
$\mathrm{C}_{26} \mathrm{H}_{35} \mathrm{~N}_{2} \mathrm{PSe}_{4}[\mathrm{M}+\mathrm{H}]: 692.8788$.

\section{1,2-Phenylenebis(methylene)}

bis $(N, N$-diisobutyl- $P$ phenylphosphonamidodiselenoate) (3i): $0.475 \mathrm{~g}$ as a pale green paste in 53\% yield. Four diastereoisomers were found in multiNMR spectra in ca. $1: 1: 3: 3$ intensity ratio. Selected IR (KBr, $\left.\mathrm{cm}^{-1}\right)$ : 1616(w), 1509(w), 1436(m), 1396(m), 1216(s), 1138(s), 1026(m), 897(s), 747(m),m 715(m), 692(s), 541(s), 487(m). ${ }^{1} \mathrm{H}$ NMR $\left(\mathrm{CD}_{2} \mathrm{Cl}_{2}, \delta\right), 8.09-7.97$ (m, 4x4H, Ar-H), 7.47-7.38 (m, 4x6H Ar-H), 7.15-7.04 (m, 4x4H, Ar-H), 4.30-3.80 (m, 4x8H, $\left.\mathrm{CH}_{2}\right)$, 2.96-2.82 (m, 4x4H, $\left.\mathrm{SeCH}_{2}\right), 1.91-1.81(\mathrm{~m}, 4 \times 4 \mathrm{H}, \mathrm{CH}), 0.85-0.75$ $\left(\mathrm{m}, 4 \times 24 \mathrm{H}, \mathrm{CH}_{3}\right) \mathrm{ppm} .{ }^{13} \mathrm{C} \mathrm{NMR}\left(\mathrm{CD}_{2} \mathrm{Cl}_{2}, \delta\right), 137.8,137.5,137.4$, 136.7, 136.4, 136.0, 135.9, 135.1, 132.2, 132.1, 131.8, 131.7, 131.0 $130.8,130.5,128.4,128.2,127.6,127.5,55.5,34.5,34.3,30.3$, 27.0, 26.9, 20.5, 20.3 ppm. ${ }^{31} \mathrm{P}$ NMR $\left(\mathrm{CD}_{2} \mathrm{Cl}_{2}, \delta\right), 77.1(\mathrm{~s}, J(\mathrm{P}, \mathrm{Se})$ $=386 \mathrm{~Hz}, J(\mathrm{P}, \mathrm{Se})=792 \mathrm{~Hz}), 76.8(\mathrm{~s}, J(\mathrm{P}, \mathrm{Se})=386 \mathrm{~Hz}, J(\mathrm{P}, \mathrm{Se})=$ $794 \mathrm{~Hz}), 76.2(\mathrm{~s}, J(\mathrm{P}, \mathrm{Se})=386 \mathrm{~Hz}, J(\mathrm{P}, \mathrm{Se})=794 \mathrm{~Hz}), 76.1(\mathrm{~s}$, $J(\mathrm{P}, \mathrm{Se})=386 \mathrm{~Hz}, J(\mathrm{P}, \mathrm{Se})=792 \mathrm{~Hz}) \mathrm{ppm} .{ }^{77} \mathrm{Se} \mathrm{NMR}\left(\mathrm{CD}_{2} \mathrm{Cl}_{2}, \delta\right)$ $357.1(\mathrm{~d}, J(\mathrm{P}, \mathrm{Se})=386 \mathrm{~Hz}), 354.1(\mathrm{~d}, J(\mathrm{P}, \mathrm{Se})=386 \mathrm{~Hz}), 350.5(\mathrm{~d}$, $J(\mathrm{P}, \mathrm{Se})=386 \mathrm{~Hz}), 350.1(\mathrm{~d}, J(\mathrm{P}, \mathrm{Se})=386 \mathrm{~Hz}),-95.6(\mathrm{~d}, J(\mathrm{P}, \mathrm{Se})=$ $792 \mathrm{~Hz}),-96.7(\mathrm{~d}, J(\mathrm{P}, \mathrm{Se})=794 \mathrm{~Hz}),-98.4(\mathrm{~d}, J(\mathrm{P}, \mathrm{Se})=794 \mathrm{~Hz})$, $-99.1(\mathrm{~d}, J(\mathrm{P}, \mathrm{Se})=792 \mathrm{~Hz}) \mathrm{ppm}$. Mass spectrum $\left[\mathrm{CI}^{+}, \mathrm{m} / \mathrm{z}\right]: 895$ $[\mathrm{M}+\mathrm{H}]^{+}$. Accurate mass measurement $\left(\mathrm{C} \mid \mathrm{I}^{+} \mathrm{MS}\right)$ : 895.0498, calculate mass for $\mathrm{C}_{36} \mathrm{H}_{55} \mathrm{~N}_{2} \mathrm{PSe}_{4}[\mathrm{M}+\mathrm{H}]:$ 895.0520.

\section{Biphenyl-4,4'-diylbis(methylene) $\quad$ bis( $N$-isopropyl- $P$ - phenylphosphonamidodiselenoate) (3j): $0.445 \mathrm{~g}$ as a reddish yellow solid in $54 \%$ yield. A pair of diastereoisomers was found in multi-NMR spectra in $c a .1: 2$ intensity ratio. Selected IR ( $\mathrm{KBr}$, $\left.\mathrm{cm}^{-1}\right)$ : 1580(w), 1495(s), 1433(m), 1396(s), 1160(s), 1094(s), 1002(m), 814(s), 741(s), 687(s), 508(s), 486(s), 409(m). ${ }^{1} \mathrm{H}$ NMR $\left(\mathrm{CD}_{2} \mathrm{Cl}_{2}, \delta\right), 8.01-7.93$ (m, 2x4H, Ar-H), 7.45-7.29 (m, 2x14H, Ar- $\mathrm{H})$, 4.20-4.17 (m, $\left.2 \times 4 \mathrm{H}, \mathrm{SeCH}_{2}\right), 3.91-3.78(\mathrm{~m}, 2 \times 2 \mathrm{H}, \mathrm{CH}), 2.65-$ $2.55(\mathrm{~m}, 2 \times 2 \mathrm{H}, \mathrm{NH}), 1.30-1.11\left(\mathrm{~m}, 2 \times 12 \mathrm{H}, \mathrm{CH}_{3}\right) \mathrm{ppm} .{ }^{13} \mathrm{C} \mathrm{NMR}$ $\left(\mathrm{CD}_{2} \mathrm{Cl}_{2}, \delta\right), 139.5,139.4,138.4,137.6,132.0,131.0,130.8,130.0$, 129.7, 129.5, 128.6, 128.4, 127.1, 126.9, 46.2, 35.7, 24.9 ppm. ${ }^{31} \mathrm{P}$ NMR $\left(\mathrm{CD}_{2} \mathrm{Cl}_{2}, \delta\right), 54.0(\mathrm{~s}, J(\mathrm{P}, \mathrm{Se})=387 \mathrm{~Hz}, J(\mathrm{P}, \mathrm{Se})=789 \mathrm{~Hz})$, $53.9(\mathrm{~s}, J(\mathrm{P}, \mathrm{Se})=387 \mathrm{~Hz}, \quad J(\mathrm{P}, \mathrm{Se})=789 \mathrm{~Hz}) \mathrm{ppm} .{ }^{77} \mathrm{Se} \mathrm{NMR}$ $\left(\mathrm{CD}_{2} \mathrm{Cl}_{2}, \delta\right), 408.5(\mathrm{~d}, J(\mathrm{P}, \mathrm{Se})=386 \mathrm{~Hz}), 408.4(\mathrm{~d}, J(\mathrm{P}, \mathrm{Se})=386$ $\mathrm{Hz}),-99.1(\mathrm{~d}, J(\mathrm{P}, \mathrm{Se})=789 \mathrm{~Hz}),-99.3(\mathrm{~d}, J(\mathrm{P}, \mathrm{Se})=789 \mathrm{~Hz}) \mathrm{ppm}$. Mass spectrum $\left[\mathrm{CI}^{+}, \mathrm{m} / \mathrm{z}\right]: 831[\mathrm{M}+\mathrm{H}]^{+}$. Accurate mass

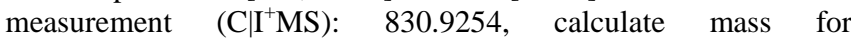 $\mathrm{C}_{32} \mathrm{H}_{39} \mathrm{~N}_{2} \mathrm{P}_{2} \mathrm{Se}_{4}[\mathrm{M}+\mathrm{H}]: 830.9257$.}

\section{1,3-Phenylenebis(methylene)}

bis $(N$-tert-butyl- $P$ phenylphosphonamidodiselenoate) (3k): $0.460 \mathrm{~g}$ as a pale yellow paste in 59\% yield. Three diastereoisomers were found in multiNMR spectra in ca. $1: 2: 2$ intensity ratio. Selected IR $\left(\mathrm{KBr}, \mathrm{cm}^{-}\right.$ $\left.{ }^{1}\right)$ : 1602(m), 1483(m), 1435(s), 1364(s), 1217(s), 1209(s), 1094(s), 993(s), 840(m), 794(m), 745(m), 691(s), 529(vs), 429(m). ${ }^{1} \mathrm{H}$ NMR $\left(\mathrm{CD}_{2} \mathrm{Cl}_{2}, \delta\right), 8.09-8.00(\mathrm{~m}, 3 \times 4 \mathrm{H}, \mathrm{Ar}-\mathrm{H}), 7.51-7.44(\mathrm{~m}, 3 \times 6 \mathrm{H}, \mathrm{Ar}-$ $\mathrm{H})$, 7.23-7.07 (m, 3x4H, Ar-H), 4.26-4.09 (m, 3x4H, $\left.\mathrm{SeCH}_{2}\right), 2.78$ (very wide, $3 \times 2 \mathrm{H}, \mathrm{NH}), 1.30-1.21\left(\mathrm{~m}, 3 \times 18 \mathrm{H}, \mathrm{CH}_{3}\right) \mathrm{ppm} .{ }^{13} \mathrm{C}$ NMR $\left(\mathrm{CD}_{2} \mathrm{Cl}_{2}, \delta\right), 140.0,139.7,139.2,139.0,138.2,137.9,132.7$, $132.2,131.8,131.1,130.9,130.5,130.4,130.3,130.1,129.9,129.1$ $128.9,128.7,128.5,128.3,128.2,128.1,127.9,127.7,56.0,55.9$, 31.4, 31.3, 27.8 ppm. ${ }^{31} \mathrm{P}$ NMR $\left(\mathrm{CD}_{2} \mathrm{Cl}_{2}, \delta\right), 46.1(\mathrm{~s}, J(\mathrm{P}, \mathrm{Se})=374$ $\mathrm{Hz}, J(\mathrm{P}, \mathrm{Se})=794 \mathrm{~Hz}), 45.9(\mathrm{~s}, J(\mathrm{P}, \mathrm{Se})=367 \mathrm{~Hz}, J(\mathrm{P}, \mathrm{Se})=789$ $\mathrm{Hz}), 45.8(\mathrm{~s}, J(\mathrm{P}, \mathrm{Se})=377 \mathrm{~Hz}, J(\mathrm{P}, \mathrm{Se})=789 \mathrm{~Hz}) \mathrm{ppm} .{ }^{77} \mathrm{Se} \mathrm{NMR}$ $\left(\mathrm{CD}_{2} \mathrm{Cl}_{2}, \delta\right), 450.7(\mathrm{~d}, J(\mathrm{P}, \mathrm{Se})=374 \mathrm{~Hz}), 447.8(\mathrm{~d}, J(\mathrm{P}, \mathrm{Se})=377$ $\mathrm{Hz}), 447.2(\mathrm{~d}, J(\mathrm{P}, \mathrm{Se})=367 \mathrm{~Hz}),-49.2(\mathrm{~d}, J(\mathrm{P}, \mathrm{Se})=789 \mathrm{~Hz}),-49.8$ $(\mathrm{d}, J(\mathrm{P}, \mathrm{Se})=789 \mathrm{~Hz}),-50.4(\mathrm{~d}, J(\mathrm{P}, \mathrm{Se})=794 \mathrm{~Hz}) \mathrm{ppm}$. Mass spectrum $\left[\mathrm{CI}^{+}, \mathrm{m} / \mathrm{z}\right]: 783[\mathrm{M}+\mathrm{H}]^{+}$. Accurate mass measurement (C|I+MS): 782.9250, calculate mass for $\mathrm{C}_{28} \mathrm{H}_{39} \mathrm{~N}_{2} \mathrm{P}_{2} \mathrm{Se}_{4}[\mathrm{M}+\mathrm{H}]$ : 782.9257 .

\section{1,2-Phenylenebis(methylene)}

bis $(N$-tert-butyl- $P$ phenylphosphonamidodiselenoate) (3l): $0.396 \mathrm{~g}$ as a greenish yellow solid in $51 \%$ yield. A pair of diastereoisomers was found in multi-NMR spectra in $c a$. $1: 1$ intensity ratio. Selected IR $(\mathrm{KBr}$, $\left.\mathrm{cm}^{-1}\right)$ : 1434(s), 1363(s), 1217(s), 1194(s), 1093(s), 990(s), 838(m), 746(m), 725(m), 688(s), 529(vs), 485(m), 432(m). ${ }^{1} \mathrm{H}$ NMR $\left(\mathrm{CD}_{2} \mathrm{Cl}_{2}, \delta\right), 8.10-8.01(\mathrm{~m}, 2 \mathrm{x} 4 \mathrm{H}, \mathrm{Ar}-\mathrm{H}), 7.49-7.44(\mathrm{~m}, 2 \mathrm{x} 6 \mathrm{H}, \mathrm{Ar}-$ $\mathrm{H})$, 7.36-7.31 (m, 2x2H, Ar-H), 7.16-7.08 (m, 2x2H, Ar-H), 4.41$4.19\left(\mathrm{~m}, 2 \mathrm{x} 4 \mathrm{H}, \mathrm{SeCH}_{2}\right), 2.83$ (wide, $\left.2 \times 2 \mathrm{H}, \mathrm{NH}\right), 1.28(\mathrm{~s}, 18 \mathrm{H}$, $\left.\mathrm{CH}_{3}\right), 1.26\left(\mathrm{~s}, 18 \mathrm{H}, \mathrm{CH}_{3}\right)$ ppm. ${ }^{13} \mathrm{C}$ NMR $\left(\mathrm{CD}_{2} \mathrm{Cl}_{2}, \delta\right), 138.4(\mathrm{~d}$, $J(\mathrm{P}, \mathrm{C})=109 \mathrm{~Hz}), 137.3(\mathrm{~d}, J(\mathrm{P}, \mathrm{C})=108 \mathrm{~Hz}), 133.3(\mathrm{~d}, J(\mathrm{P}, \mathrm{C})=$ $3.1 \mathrm{~Hz}), 131.8(\mathrm{~d}, J(\mathrm{P}, \mathrm{C})=3.1 \mathrm{~Hz}), 131.2,131.0,130.8,130.4$, $128.5,128.4,128.3,127.9,127.7,127.6,56.0,55.9,34.5,31.4$, 31.3 ppm. ${ }^{31} \mathrm{P}$ NMR $\left(\mathrm{CD}_{2} \mathrm{Cl}_{2}, \delta\right), 46.0(\mathrm{~s}, J(\mathrm{P}, \mathrm{Se})=376 \mathrm{~Hz}$, $J(\mathrm{P}, \mathrm{Se})=789 \mathrm{~Hz}), 45.9(\mathrm{~s}, J(\mathrm{P}, \mathrm{Se})=378 \mathrm{~Hz}, J(\mathrm{P}, \mathrm{Se})=786 \mathrm{~Hz})$ ppm. ${ }^{77} \mathrm{Se} \mathrm{NMR}\left(\mathrm{CD}_{2} \mathrm{Cl}_{2}, \delta\right), 438.8(\mathrm{~d}, J(\mathrm{P}, \mathrm{Se})=378 \mathrm{~Hz}), 438.1(\mathrm{~d}$, $J(\mathrm{P}, \mathrm{Se})=376 \mathrm{~Hz}),-50.3(\mathrm{~d}, J(\mathrm{P}, \mathrm{Se})=789 \mathrm{~Hz}),-50.4(\mathrm{~d}, J(\mathrm{P}, \mathrm{Se})=$ $789 \mathrm{~Hz}) \mathrm{ppm}$. Mass spectrum $\left[\mathrm{CI}^{+}, \mathrm{m} / \mathrm{z}\right]: 783[\mathrm{M}+\mathrm{H}]^{+}$. Accurate mass measurement $\left(\mathrm{C} \mid \mathrm{I}^{+} \mathrm{MS}\right)$ : 782.9238, calculate mass for $\mathrm{C}_{28} \mathrm{H}_{39} \mathrm{~N}_{2} \mathrm{P}_{2} \mathrm{Se}_{4}[\mathrm{M}+\mathrm{H}]: 782.9257$.

\section{1,2-Phenylenebis(methylene)}

bis( $N$-sec-butyl- $P$ phenylphosphonamidodiselenoate) (3m): $0.420 \mathrm{~g}$ as a yellow paste in $54 \%$ yield. Four diastereoisomers were found in multiNMR spectra in ca. $1.0: 1.0: 1.2: 1.0$ intensity ratio. Selected IR $\left(\mathrm{KBr}, \mathrm{cm}^{-1}\right):$ 1453(m), 1434(s), 1398(m), 1131(m), 1096(s), 1038(m), 1007(m), 949(m), 852(m), 744(s), 688(s), 534(vs), 490(m). ${ }^{1} \mathrm{H}$ NMR $\left(\mathrm{CD}_{2} \mathrm{Cl}_{2}, \delta\right), 8.15-7.90(\mathrm{~m}, 4 \times 4 \mathrm{H}, \mathrm{Ar}-\mathrm{H}), 7.56-$ $7.30(\mathrm{~m}, 4 \mathrm{x} 6 \mathrm{H}, \mathrm{Ar}-\mathrm{H}), 7.27-7.05(\mathrm{~m}, 4 \mathrm{x} 4 \mathrm{H}, \mathrm{Ar}-\mathrm{H}), 4.23-4.05(\mathrm{~m}$, $\left.4 \times 4 \mathrm{H}, \mathrm{SeCH}_{2}\right), 3.95-3.86(\mathrm{~m}, 4 \times 2 \mathrm{H}, \mathrm{CH}), 2.69-2.66(\mathrm{~m}, 4 \times 2 \mathrm{H}$, $\mathrm{NH}), 1.62-1.28\left(\mathrm{~m}, 4 \times 4 \mathrm{H}, \mathrm{CH}_{2}\right), 1.15-1.09\left(\mathrm{~m}, 4 \times 6 \mathrm{H}, \mathrm{CH}_{3}\right), 0.85$ $0.83\left(\mathrm{~m}, 4 \times 6 \mathrm{H}, \mathrm{CH}_{3}\right)$ ppm. ${ }^{13} \mathrm{C} \mathrm{NMR}\left(\mathrm{CD}_{2} \mathrm{Cl}_{2}, \delta\right), 137.6,137.2$, 136.5, 135.9, 133.3, 132.0, 131.7, 131.1, 130.9, 130.5, 128.6, 128.4, 127.8, 127.7, 51.3, 51.2, 33.9, 32.4, 31.5, 30.2, 22.0, 10.2 ppm. ${ }^{31} \mathrm{P}$ $\operatorname{NMR}\left(\mathrm{CD}_{2} \mathrm{Cl}_{2}, \delta\right), 55.2(\mathrm{~s}, J(\mathrm{P}, \mathrm{Se})=384 \mathrm{~Hz}, J(\mathrm{P}, \mathrm{Se})=789 \mathrm{~Hz})$, $55.1(\mathrm{~s}, J(\mathrm{P}, \mathrm{Se})=384 \mathrm{~Hz}, J(\mathrm{P}, \mathrm{Se})=789 \mathrm{~Hz}), 54.8(\mathrm{~s}, J(\mathrm{P}, \mathrm{Se})=$ $384 \mathrm{~Hz}, J(\mathrm{P}, \mathrm{Se})=789 \mathrm{~Hz}), 54.7(\mathrm{~s}, J(\mathrm{P}, \mathrm{Se})=387 \mathrm{~Hz}, J(\mathrm{P}, \mathrm{Se})=$ $789 \mathrm{~Hz}) \mathrm{ppm} .{ }^{77} \mathrm{Se} \mathrm{NMR}\left(\mathrm{CD}_{2} \mathrm{Cl}_{2}, \delta\right), 395.9(\mathrm{~d}, J(\mathrm{P}, \mathrm{Se})=384 \mathrm{~Hz})$, $395.2(\mathrm{~d}, J(\mathrm{P}, \mathrm{Se})=384 \mathrm{~Hz}), 394.8(\mathrm{~d}, J(\mathrm{P}, \mathrm{Se})=384 \mathrm{~Hz}), 391.4(\mathrm{~d}$, $J(\mathrm{P}, \mathrm{Se})=387 \mathrm{~Hz}),-99.0(\mathrm{~d}, J(\mathrm{P}, \mathrm{Se})=789 \mathrm{~Hz}),-99.5(\mathrm{~d}, J(\mathrm{P}, \mathrm{Se})=$ $789 \mathrm{~Hz}),-101.1(\mathrm{~d}, J(\mathrm{P}, \mathrm{Se})=789 \mathrm{~Hz}),-101.4(\mathrm{~d}, J(\mathrm{P}, \mathrm{Se})=789 \mathrm{~Hz})$ ppm. Mass spectrum $\left[\mathrm{CI}^{+}, \mathrm{m} / \mathrm{z}\right]: 783[\mathrm{M}+\mathrm{H}]^{+}$. Accurate mass measurement $\left(\mathrm{C} \mid \mathrm{I}^{+} \mathrm{MS}\right)$ : 782.9249, calculate mass for $\mathrm{C}_{28} \mathrm{H}_{39} \mathrm{~N}_{2} \mathrm{P}_{2} \mathrm{Se}_{4}[\mathrm{M}+\mathrm{H}]: 782.9257$.

\section{1,3-Phenylenebis(methylene)}

bis $(N$-sec-butyl- $P$ phenylphosphonamidodiselenoate) (3n): $0.470 \mathrm{~g}$ as a yellow paste in $60 \%$ yield. Four diastereoisomers were found in multiNMR spectra in ca. $1.1: 1.0: 1.0: 1.2$ intensity ratio. Selected IR $\left(\mathrm{KBr}, \mathrm{cm}^{-1}\right): 1602(\mathrm{~m}), 1485(\mathrm{~m}), 1435(\mathrm{~s}), 1396(\mathrm{~m}), 1184(\mathrm{~m})$ 1133(m), 1097(s), 1-38(m), 1008(m), 951(m), 744(s), 692(vs), 535(vs), 487(m). ${ }^{1} \mathrm{H}$ NMR $\left(\mathrm{CD}_{2} \mathrm{Cl}_{2}, \delta\right), 8.15-7.96$ (m, 4x4H, Ar-H), 7.51-7.46 (m, 4x6H, Ar-H), 7.14-6.90 (m, 4x4H, Ar-H), 4.10-4.04 (m, $4 \times 4 \mathrm{H}, \mathrm{SeCH}_{2}$ ), 3.82-3.61 (m, 4x2H, CH), 2.69-2.64 (m, 4x2H, $\mathrm{NH}), 1.45-1.38\left(\mathrm{~m}, 4 \times 4 \mathrm{H}, \mathrm{CH}_{2}\right), 1.19-1.10\left(\mathrm{~m}, 4 \times 6 \mathrm{H}, \mathrm{CH}_{3}\right), 0.98-$ $0.83\left(\mathrm{~m}, 4 \times 6 \mathrm{H}, \mathrm{CH}_{3}\right)$ ppm. ${ }^{13} \mathrm{C}$ NMR $\left(\mathrm{CD}_{2} \mathrm{Cl}_{2}, \delta\right), 139.7,138.7$, 137.1, 135.9, 132.1, 131.1, 130.9, 130.2, 130.0, 129.8, 128.9, 128.6, 128.4, 128.0, 127.9, 127.6, 51.3, 51.1, 50.1, 36.1, 35.9, 32.4, 31.5, 28.0, 27.4, 22.0, 18.2, 10.2 ppm. ${ }^{31} \mathrm{P}$ NMR $\left(\mathrm{CD}_{2} \mathrm{Cl}_{2}, \delta\right), 55.0(\mathrm{~s}$ $J(\mathrm{P}, \mathrm{Se})=390 \mathrm{~Hz}, J(\mathrm{P}, \mathrm{Se})=789 \mathrm{~Hz}), 54.9(\mathrm{~s}, J(\mathrm{P}, \mathrm{Se})=390 \mathrm{~Hz}$, $J(\mathrm{P}, \mathrm{Se})=789 \mathrm{~Hz}), 54.6(\mathrm{~s}, J(\mathrm{P}, \mathrm{Se})=390 \mathrm{~Hz}, J(\mathrm{P}, \mathrm{Se})=789 \mathrm{~Hz})$, $54.5(\mathrm{~s}, J(\mathrm{P}, \mathrm{Se})=390 \mathrm{~Hz}, \quad J(\mathrm{P}, \mathrm{Se})=789 \mathrm{~Hz}) \mathrm{ppm} .{ }^{77} \mathrm{Se} \mathrm{NMR}$ $\left(\mathrm{CD}_{2} \mathrm{Cl}_{2}, \delta\right), 406.4(\mathrm{~d}, J(\mathrm{P}, \mathrm{Se})=390 \mathrm{~Hz}), 406.2(\mathrm{~d}, J(\mathrm{P}, \mathrm{Se})=390$ $\mathrm{Hz}), 406.1(\mathrm{~d}, J(\mathrm{P}, \mathrm{Se})=390 \mathrm{~Hz}), 405.7(\mathrm{~d}, J(\mathrm{P}, \mathrm{Se})=390 \mathrm{~Hz})$, $97.1(\mathrm{~d}, J(\mathrm{P}, \mathrm{Se})=789 \mathrm{~Hz}),-97.5(\mathrm{~d}, J(\mathrm{P}, \mathrm{Se})=789 \mathrm{~Hz}),-99.1(\mathrm{~d}$, $J(\mathrm{P}, \mathrm{Se})=789 \mathrm{~Hz}),-99.5(\mathrm{~d}, J(\mathrm{P}, \mathrm{Se})=789 \mathrm{~Hz}) \mathrm{ppm}$. Mass spectrum $\left[\mathrm{CI}^{+}, \mathrm{m} / \mathrm{z}\right]: 783[\mathrm{M}+\mathrm{H}]^{+}$. Accurate mass measurement (C|I+MS): 782.9246, calculate mass for $\mathrm{C}_{28} \mathrm{H}_{39} \mathrm{~N}_{2} \mathrm{P}_{2} \mathrm{Se}_{4}[\mathrm{M}+\mathrm{H}]$ : 782.9257 . 


\section{Acknowledgments}

The authors are grateful to the University of St Andrews for financial support and the EPSRC National Mass Spectrometry Service Centre (Swansea) for mass spectral measurements.

[1] R. A. Cherkasov, G. A. Kutyrev, A. N. Pudovik, Tetrahedron 1985, 41, 2567-2624.

[2] F. H. Westheimer, Science 1987, 235, 1173-1178.

[3] L. D. Quin, A Guide to Organophosphorus Chemistry; Wiley Interscience: New York, 2000.

[4] V. D. Warner, D. B. Mirth, A. S. Dey, J. Med. Chem. 1973, 16, 1185-1186.

[5] R. F. Borch, G. W. Canute, J. Med. Chem. 1991, 34, 30443052 .

[6] Z. R. Li, J. Y. Han, Y. Y. Jiang, P. Browne, R. J. Knox, L. Q. Hu, Bioorg. Med. Chem. 2003, 11, 4171-4178.

[7] S. V. Levchik, E. D. Weil, Polym. Int. 2005, 54, 11-35.

[8] M. Mevel, T. Montier, F. Lamarche, P. Delepine, T. Le Gall, J. J. Yaouanc, P. A. Jaffres, D. Cartier, P. Lehn, J. C. Clement, Bioconjugate Chem. 2007, 18, 1604-1611.

[9] G. Keglevich, A. Kerenyi, Trends Org. Chem. 2008, 12, 7377.

[10] L. Kollar, G. Keglevich, Chem. Rev. 2010, 110, 4257-4302.

[11] I. P. Gray, P. Bhattacharyya, A. M. Z. Slawin, J. D. Woollins, Chem. Eur. J. 2005, 11, 6221-6227.

[12] For reviews, see a) G. Hua, J. D. Woollins. Angew. Chem. Int. Ed. 2009, 48, 1368-1377; b) J. D. Woollins, Synlett 2012, 1154-1169.

[13] G. Hua, J. B. Henry, Y. Li, A. R. Mount, A. M. Z. Slawin, J. D. Woollins, Org. Biomol. Chem. 2010, 8, 1655-1660.

[14] G. Hua, Y. Li, A. L. Fuller, A. M. Z. Slawin, J. D. Woollins, Eur. J. Org. Chem. 2009, 1612-1618.

[15] G. Hua, A. L. Fuller, Y. Li, A. M. Z. Slawin, J. D. Woollins, New J. Chem. 2010, 34, 1565-1571.

[16] G. Hua, A. L. Fuller, A. M. Z. Slawin, J. D. Woollins, Eur. J. Org. Chem. 2010, 2707-2615.

[17] G. Hua, A. L.Fuller, A. M. Z. Slawin, J. D. Woollins, Polyhedron 2011, 30, 805-808.

[18] G. Hua, A. L.Fuller, A. M. Z. Slawin, J. D. Woollins, Eur. J. Org. Chem. 2011, 3067-3073.

[19] G. Hua, J. M. Griffin, S. E. Ashbrook, A. M. Z. Slawin, J. D. Woollins, Angew. Chem. Int. Ed. 2011, 50, 4123-4126.

[20] G. Hua. D. B. Cordes, Y. Li, A. M. Z. Slawin, J. D. Woollins, Tetrahedron Lett. 2011, 52, 3311-3314.

[21] G. Hua, A. L. Fuller, A. M. Z. Slawin, J. D. Woollins, Synlett 2012, 23, 2453-2458.

[22] G. Hua, J. D. Woollins, Polyhedron 2012, 42, 190-195.

[23] G. Hua, A. M. Z. Slawin, J. D. Woollin, Synlett 2012, 23, 1170-1174.

[24] G. Hua, A. L. Fuller. A. M. Z. Slawin, J. D. Woollins, Synlett 2012, 23, 2453-2458.

[25] G. Hua, R. A. M. Randall, A. M. Z. Slawin, D. B. Cordes, L. Crawford, M. Bühl, J. D. Woollins, Chem. Commun. 2013, 49, 2619-2621.

[26] G. Hua, R. A. M. Randall, A. M. Z. Slawin,J. D. Woollins, Z. Anorg. Allg. Chem. 2011, 637, 1800-1806.

[27] G. Hua, R. A. M. Randall, A. M. Z. Slawin, J. D. Woollins, Tetrahedron 2013, 69, 5299-5305.

[28] Crystallographic data for compound $2 e . \mathrm{C}_{18} \mathrm{H}_{20} \mathrm{BrNOPSe}_{2}$, $M=535.16$, Triclinic, space group $P-1, a=8.176(8) \AA$, $b=10.242(10) \AA, c=12.652(13) \AA, \alpha=81.19(3)^{\circ}, \beta=$ $79.35(3)^{\circ}, \gamma=75.78(3)^{\circ}, U=999.6(17) \AA^{3}, Z=2, \mu=$ $5.7896 \mathrm{~mm}^{-1}, 8488$ reflections, 3516 unique $\left(R_{\text {int }}=0.0827\right)$; $R_{1}=0.0522, w R_{2}=0.1329$.

[29] Crystallographic data for compound $2 i$. $\mathrm{C}_{19} \mathrm{H}_{20} \mathrm{BrNOPSe}_{2}, M$ $=547.17$, Monoclinic, space group $P 2_{1 / c}, a=6.956(6) \AA$, $b=17.86(2) \AA, c=32.53(3) \AA, \beta=92.038(11)^{\circ}, U=$ 4040(6) $\AA^{3}, Z=8, \mu=5.7318 \mathrm{~mm}^{-1}, 33067$ reflections,
7116 unique $\left(R_{\text {int }}=0.01636\right) ; R_{1}=0.0659, w R_{2}=0.1445$

[30] P. Bhattacharyya, A. M. Z. Slawin, J. D. Woollins, J. Organomet. Chem. 2001, 623, 116-119.

[31] a) P. Bhattacharyya, A. M. Z. Slawin, J. D. Woollins, Chem Eur. J. 2002, 8, 2705-2711; b) P. Bhattacharyya, A. M. Z. Slawin, J. D. Woollins, Dalton Trans. 2001, 300-303; c) P. Bhattacharyya, J. Novosad, J. R. Phillips, A. M. Z. Slawin, D. J. Williams, J. D. Woollins, Dalton Trans. 1995, 16071613.

[32] G. Hua, Q. Zhang, Y. Li, A. M. Z. Slawin, J. D. Woollins, Tetrahedron 2009, 65, 6074-6082.

[33] P. Kilian, P. Bhattacharyya, A. M. Z. Slawin, J. D. Woollins, Eur. J. Inorg. Chem. 2003, 1461-1467.

[34] K. Karaghiosoff, K. Eckstein, Phosphorus, Sulfur Silicon Relat. Elem. 1993, 75, 257-260.

[35] M. J. Pilkington, A. M. Z. Slawin, D. J. William, D. J. Woollins, Heteroatom Chem. 1990, 1, 351-355.

[36] G. M. Sheldrick, SHELXL97. Acta Cryst. 2008, A64, 112122.

Received: ((will be filled in by the editorial staff)) Published online: ((will be filled in by the editorial staff) 


\section{Entry for the Table of Contents}

Woollins' reagent has been applied as a highly efficient building block or unit for the synthesis of organo seleniumphosphorus heteroatom compounds.
Organo Phosphorus-Selenium Chemistry

Guoxiong Hua, Kasun S. A. Arachchige, Alexandra M. Z. Slawin, nd J. Derek Woollins*....... Page No. ... Page No.

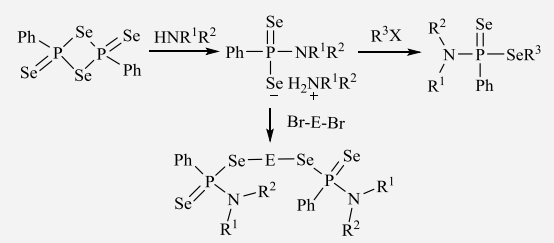

Organo Phosphorus-Selenium

Heteroatom Derivatives

from Selenation of Primary/Secondary Amines and Haloalkanes/Dihaloalkanes

Keywords: Woollins' reagent / Selenation / Phosphorus-Selenium Compounds / Amines / Haloalkanes 